P $\quad$ R A A T I C H E

\title{
OSSER VAZIONI
}

INTORNOAL GOVERNO

D E' C A V A L L E R I, EALLA COLTIVAZ1ONE

\section{DE' MORARI, VITI, E SIEPI,}

NEI TERRENI MAGRI, E SASSOSI

\section{Del Territorio Veronese.}

ED IN QUESTA SECONDA EDIZIONE AGGIUNTEVENE DALL'AUTORE ALCUNE ALTRE UTI. LISSIME INTORNO LE API.

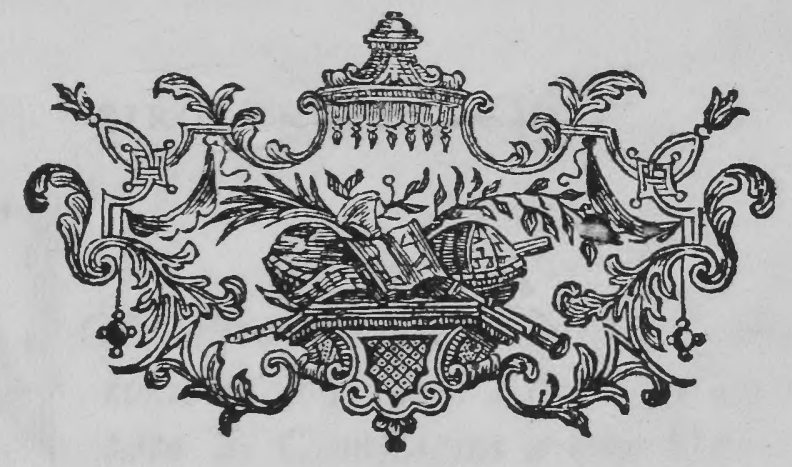

I N VERONA, MDCCLXI.

Per Agoftino Carattoni Stampator Vefcovile. CON LICENZA DE' SUPERIORI. 


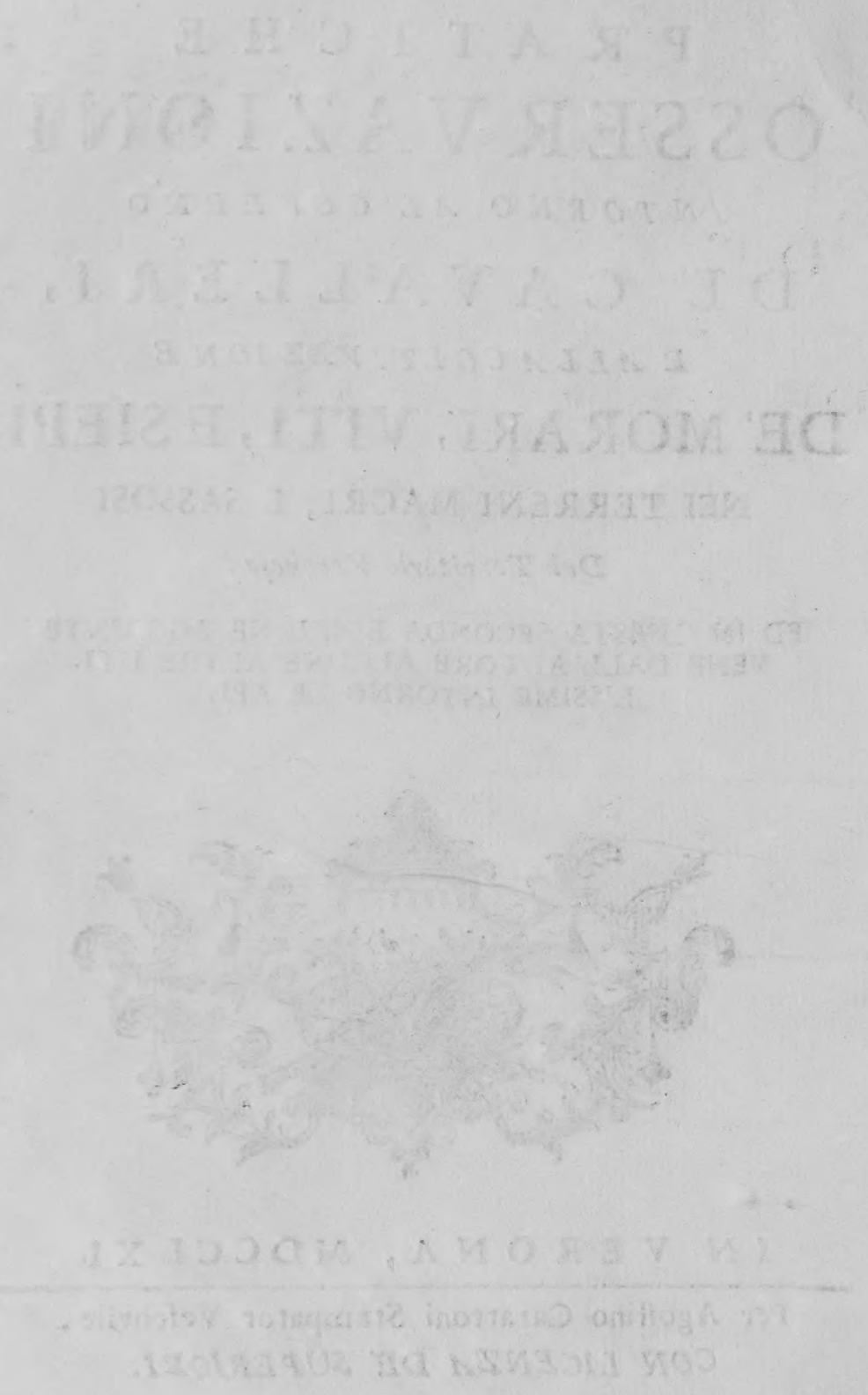


ALLA NOBIL SIGNORA CONTESSA

\section{A SSIM ILI A N A DE GUARIENTI GAZOLA}

GIROLAMO MARANI.

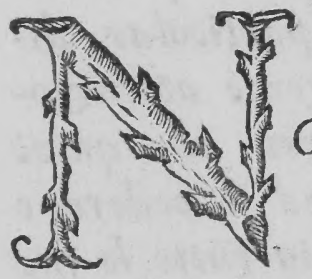

ON deve far meraviglia ad alcuno; come io $m$ 'avanzi a dedicare un Trattato di Campagna a una Dama, cbe alla coltura non delle Campagne, ma delle lettere $e$ delle piiu nobili arti di continuo attende. E qua. le è mai quello ftudia, Nobil Signora Conteffa, a cui non fi applicbi la Jua bella mente, e non ne A 2 riefca 
riefca in tutti con fomma lode, e con gran lume di fcienza non ne ragioni? Lo Atudio dell' Agri. coltura, come non è già l'ultimo fra gli altri nel pregio, così non è efclue dal juo virtuofo genio, $e$ dal $\int u o$ vivaciffimo spirito e prudentiffimo. Ol. tre all' iftinto naturale, cbe porta feco dalla $\mathrm{N}_{a}$ zion Alemanna, delle arti e fcienze coltivatrice 0 perofa, e dell' Agricoltura fingolarmente, lode propria $f_{i}$ è quefta del di lei raro ingegno e più che virile talento, che tutto penetra, tutto ricerca, $a$ tutto abbraccia e comprende, quanto è degno a $\int a$. perfi da una Dama compita, qual' Ella ̀̀, $e$ inde. feffa non men negli Audj, che negl' impiegbi. Ed ecco al propofito mio una ragion più precifa del do. ver farle quefta offequiofifima mia Offerta. Tra i molti fertili Poderi, che la Nobil Ca a G AZOLA in quefto noftro Territorio poffede, uno ne gade appunto in luogo fimile a quello di cui qui fi trat. ta, nel qual più cbe in altri Ella fi compiace $a^{*}$ juoi tempi di Joggiornare; e un $\int u o$ particolar diletto fuol effere l'adoperarfi con diligente attenzione fopra il buon governo dei Cavalleri, che quivi in grande albondanza fi tengono; ed il vedere $e$ provvedere, che fia quel vafto jiabile in tutte le fue parti ben coltivato. Qual coja per tanio piis ragionevole, che, avendo io fatte alcun: utili Offerva. zioni in un terreno di fimil natura, cbe alla mia 
povera cafa appartiene, e volendo io darle al pub. blico, per farne comune l'utilita, ad una tal infigne Dama mia Padrona presentar le dove si, affine di metterle prima fotto ai riflejsi benigni del-

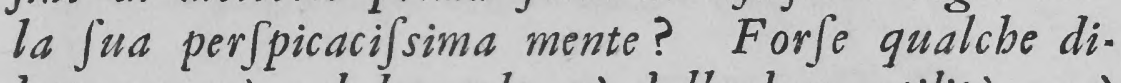
letto trarrà nel leggerle, si dalla loro utilità, e sì ancora dalla Jemplicita, con cui fono efpreffe. Se non altro fi degnera aggradire nella tenuita e rozzezza del dono il buon animo e profondifsimo offequio dell' Offerente. E cbi Sa, cbe non mi donaf$\int e$ ancbe l'onore di ordinare un qualcbe lavoro nelle Jue terre a norma di quanto bo qui fcritto? Lo che fe giungo ad ottenere, oltre modo onorato mi riputarò dalla bontà e gentilezza fua; e non avrò altro a bramare con ogni mia pofsibile opera, cbe di mantenermi nella jua pregiatifsima grazia; cui per fine umilifsimamente mi raccomando. 


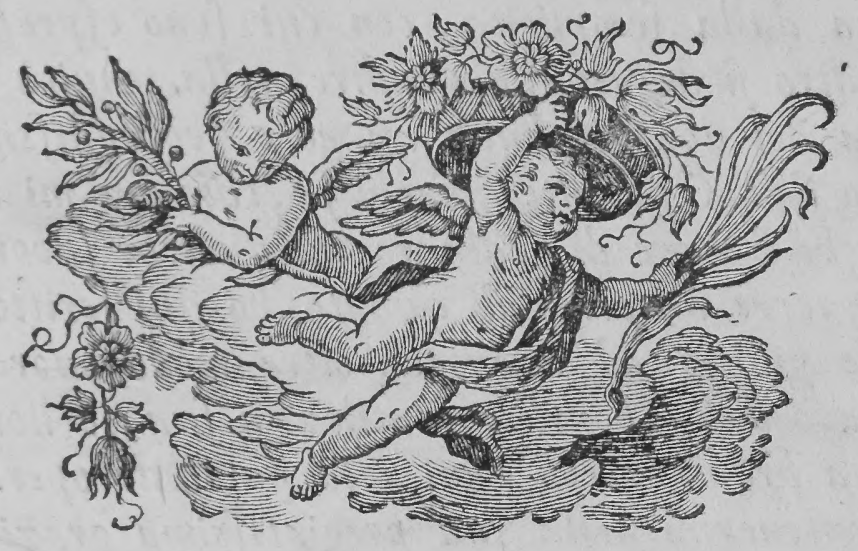



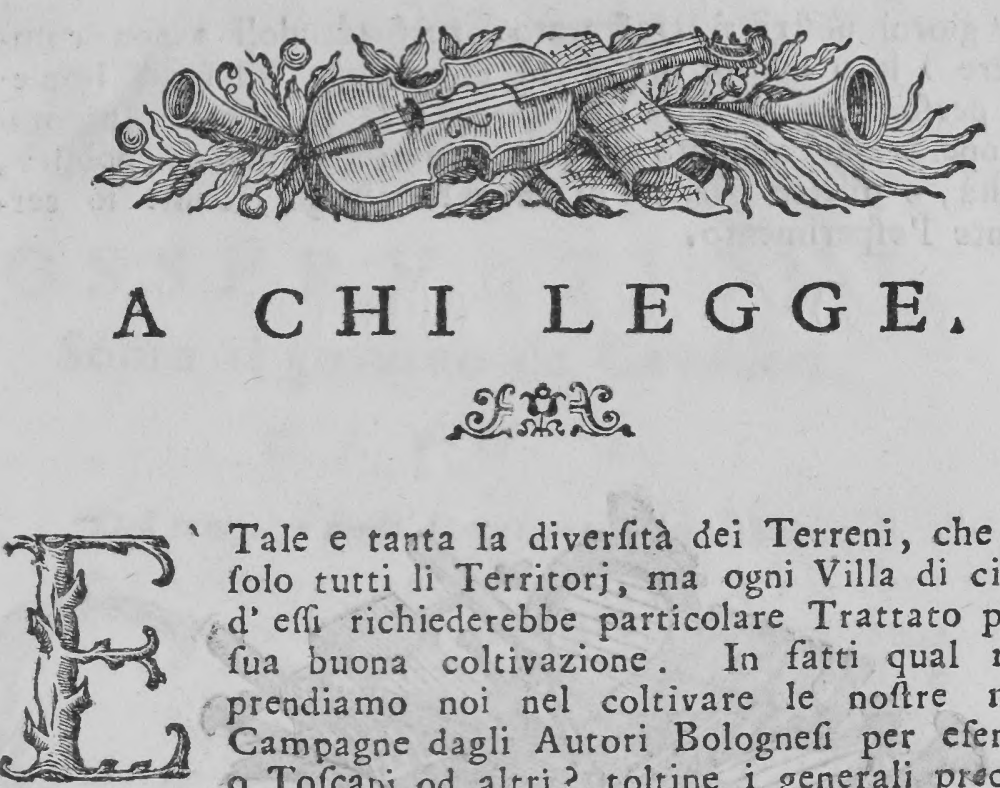

Tale e tanta la diverfità dei Terreni, che; non folo tutti li Territori, ma ogni Villa di ciafcun d'effi richiederebbe particolare Trattato per la fua buona coltivazione. In fatti qual regola prendiamo noi nel coltivare le noftre magre Campagne dagli Autori Bolognefi per efempio, - Tofcani od altri? toltine i generali precetti, appena alcuna. Tuttavia non intendo qui di fare un Trattato di Agricoltura per le noftre Campagne Veronefi, che a tanto non giunge il mio poco fapere: lafcio quefta imprefa a più nobili e follevati intelletti, de' quali a meraviglia abbonda oramai quefta noftra Patria Solamente per zelo del pubblico bene, e vantaggio de miei Concittadini met. to in vifta un picciolo compleflo di alcune mie offervazioni rurali, fondate fu la propria fperienza. Non intendo di dare nè meno tutte le regole delle materie efpofte, ma folo le più neceffarie, e le più minute ancora, come quelle, che più facilmente trafcuranfi, e che forfe maggior utile apportano, e le più proprie des terreni magri e faffofi, de' quali la Divina Provvidenza $f_{1}$ è degnata di compartirne liberalmente alla mia Cafa. Bramo, che fervano quefte regole tanto ai Villici fteffi, quanto ai Padroni: e per tale effetro mi fervo di termini ufuali e comuni, per rendermi intelligibile anche ai più idioti. Vorrei per altro, che qualche più erudita penna e colta forgeffe ad innamorare i Padroni in quefto ftu. 
dio a' giorni noftri sì trafcurato; perfuadendoli a non com. mettere i loro campi all' alcrui difcrezione, ma ad impiegarfi perfonalmente nella foprantendenza di qualche premurofa operazione nei loro Poderi, commendando la nobiltà, l'utilità, e diletto grande di un tal impiega, come io certamente l'efperimento.

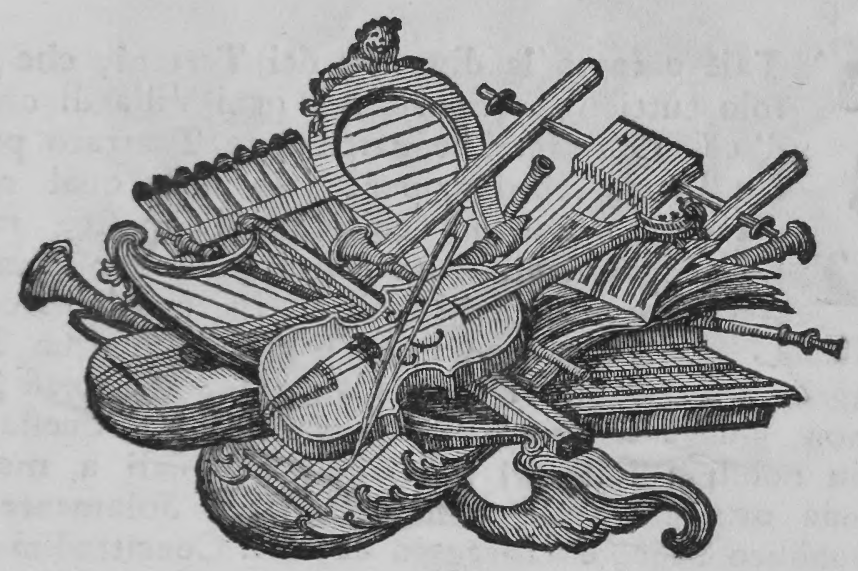




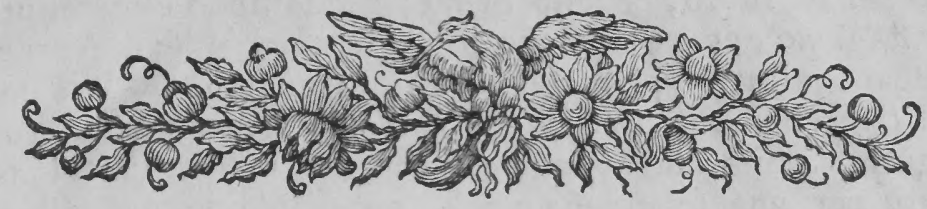 \\ O S S R V A Z I O N I}

Sopra il governo de' Cavalleri.

\section{$\begin{array}{llllll}C & A & \text { P } & O & \text { I. }\end{array}$}

Del tempo, e modo di mettere i Cavalleri.

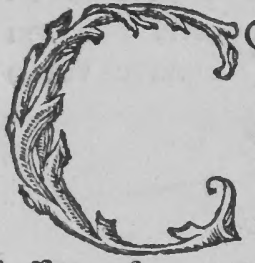

Orre detto comune tra' Villici, che il buon efito de' Cavalleri è gioco di fortuna, e non effere ancora nato colui, che abbia imparato la maniera di allevarli; ma ciò credo un' inganno, anzi un mezzo termileffero fare a chi vanno male; mentre offervo per efperienza non effer cofa sì facile, che male riefcano ai dir ligenti offervatori delle buone regole, tanto quanto ai negligenti. Io fon beniffimo perfuafo, coll opinione di molti, che fe veniffero da' Contadini offervate le debite regole nel farli nafcere, nell allevarli, nel mantenerli, nel porli al lavoro, et ordinare i bofchi, nello fcoprirli e far la femenza, maggior quantità fi farebbe di feta, di quel ch'ora fi faccia; delle quali core ad una ad una anderò parlando.

Premeffa dunque la benedizione della femenza, ed imploratone da Sua Divina Maeftà l'effetto per tale importante entrata; quanto al metterli dirò: Non è forfe vero che fe nafcono male, ordinariamente parlando, riefcono anche male nel decorfo della lor vita? non abbiano pertanto troppo fretta le Donne nel farli na. fcere; ma vadino a tempo, e con caldo naturale fenza menza. 
Io

Opervazioni

fervirfi di fuoco, o delle Stuffe; procurando che vénuta la ftagione opportuna, che fuol effere nel Mere d'Aprile, nafcano almeno prima dell' ultimo quarto della Luna col fine che vadino al lavoro entro la Luna vecchia fuf. feguente. Si vada dunque difponendo la fernenza al na. fcere per una fettimana e più, ponendola in pannolino; avvertendo di porne poca quantità per fazzolerto e difte. fa, quando fi mette a covare, acciò nafcano fani, vigo. rofi ed eguali. Configlierei ancora a far foccede picciole; oppure fe grandi, mettere la femenza in due tempi, qual. ora la ftagione non dia fretta, come nell' anno corrente; cioè colla diftanza di almeno tre giorni da una muta all' altra, per dar facilità a' Villici di ufarne il do. vuto governo nel fine, mentre promettono molto, ma delle fan attendono poco, maffimamente in quefto particolare. ฉe.

Le ftanze per li Cavalleri fiano afciutte, perchè poi non divengano Calcinaroli. Quelle che fono fituate verfo Levante e Ponente fono le migliori.

\section{A P O I I.}

Del modo di allevarli.

Uanto all allevarli ognuno sà, che ful principio devefi fervire delle foglie più tenere e più piccio1e. Nati che fono i Cavalleri, fi levano dalla femen. $z a$, ftando in luogo caldo al fuoco, e meglio al fole, purchè fi tengano coperti in maniera, che fentano il calore, ma raggio folare non li tocchi; ponendo fopra la femenza già nata primieramente o una Carta forata a foggia di Crivello, oppure certa Erba ben nota, detta volgarmente Danea, e poi fopra quella o quefta le foglie piccole e tenere di Moraro; acciò formontando all' odore delle medefime i foli Cavalleri nati, reftino fotto la Carta o Erba fuddetta i femi da nafcere. Allorchè fono formontati fi levano e fi pongano fopra fogli di carta groffa, ftroppicciati o fia fregati prima con erbe odorifere, e fi portano vicini al fuoco in luogo caldo 


\section{Soprá il governo de' Cavalleri.}

già preparato, e difefo dall' aria. E perchè per lo più nafcono in due o tre giorni, così fi deve ufar diligenza nel tener feparati quei del primo giorno da quei del fecondo, e fuffeguentemente tanto fopra le carte, quan. aione feto fopra i tavolini, quando coll' andar del tempo non età.

fi raggiungeffero, come fpeffe volte fuccede. Talvolta avviene che da piccioli per la lor debolezza reftano fotto la foglia e fi perdono; in tal cafo è neceffario trafportar di frequente in altri fogli quelli che fon venuti al di fopra, ajutando con nuova foglia gli altri ad afcendere.

Si mutano i Cavalleri immediatamente avanti e do. po le dormite, levandoli deftramente, e non ripiegandoli a rotolo per non nuocerli, e ponendo i letti a par-. te per raccoglier con foglia fparfavi fopra quelli che Mododi vi reftano dentro. Quando fono delle quattro, fi mutano ogn' altro giorno; e piì fpeffo, s'è tempo caldo, netrando fempre i tavoloni con erbe odorofe. Si avver. te che nel mutarli la prima volta dopo le dormite, fi trafportino in altro fito quei che non fono levati, gettando via $i$ guafti acciò non infettino i fani. Per tali riguardi farebbe più utile e ficura regola il fervirfi delle reti, come ufano i Trentini, i quali ogni volta che Ujo Trenmutano i Cavalleri principiando dalla dormita delle tre, tino. - almeno delle quattro, ftendono fopra i tavoloni una rete fatca a tal uro, dandovi fopra il fuo pafto di foglia; e frattanto che i Cavalleri formontano, atraccano la rete fuddetta d'intorno al ravolone di fopra, alzando il quale refta in aria la rete co' Cavalleri: quindi hanno pronto il comodo di trasferire quelli che ancora dormono, e di nettare i tavoloni Ciò fatto, calano la rete, e refano ivi i Cavalleri netti, egual, e fenza pericolo che fiano offefi: E ficcome in tal modo rimangono obbligate le reti fotto a' Cavalleri, ne ri. fervano una fempre in libertà per fervirfene fucceflivamente all' ifteffa maniera.

Riguardo al fuoco, alcuni fono d'opinione che male fia ufarlo; l'efperienza però ne infegna tutto il contrario, 


\section{Ofjervazioni}

Uso del rio, mentre a vero dire dal fuoco ufato in buona for: ma dipende in miglior parte l' efito de' Cavalleri, accellerandone quefto la vita, e rendendone più breve e meno pericolofa l' infermità delle dormite; e perciò più vegeti riufcendo, maggior quantità e qualità miglior Regola di galette producono. La regola dunque del fuoco fia quedelfuoco. fta. Si procuri di far afcendere col fuoco l'aere delle Camere dove fi nutrifcono i Cavalleri nel principio della lor vita a quel grado di caldo, a cui nel fine afcenderà col benefizio della ftagione, moderandolo nel progreffo conforme avanzerà il caldo naturale. Si of. fervi pero che la mattina, la fera, e la notte fa più frefco ordinariamente che nel decorfo del giorno; così ancora fuccedono alcuni giorni più freddi a cagion di pioggia o di vento; onde in fimili cafi ove manca naUso del tura, arte fupplifca. Piacemi perciò l' ufo del termosersome-metro da alcuni introdotto con fommo vantaggio, il tro. quale appuntato al grado temperato più o meno, giufta la cognizione di chi governa i Cavalleri, al medefimo grado col fuoco fi mantiene fino al fine, accrefcendo, diminuendo, oppure levando affatto il fuoco fecondo il bifogno. E' neceffario anche introdurre di quando in quando nei luoghi de' Cavalleri qualche tratto d'aria temperata allorache il tempo è placido affin di purgarli da qualunque mal odore e confervarli fani.

Prima che i Cavalleri dormano delle quattro, gli ine-

Altra guali di età fi feparino anco di camera, rari elfendo n. quegli anni, nei quali fogliati delle quattro non abbiano bifogno di aere più che di caldo; ficchè non potendoli fogliar tutti nel medefimo tempo per la differenza dell' età, fi rende neceffaria tal feparazione per mantenerli tutti nella loro aria competente, cioè i fogliati nella temperata, e quelli da fogliare nel caldo pur temperato. Si guardino in tal tempo dal caldo, mafimamente nella notte, che più facilmente per il frefco efteriore fi ferra nelle cafe, come fuccede anche ne' giorni nuvolofi. Giova ancora temperare l' umidità della mattina e della fera col fumo de' lettiabbrucciati 
Sopra il governo de' Cavalleri.

ciati al di fuori delle cafe. Si avverte per ultimo di Dalfuriguardarli fempre con gelofia dalli ftrepiti e cattivi $i_{t i}^{\text {mostarepi. }}$ odori odori, come pure dal lafciarvi fopra cadere oglio percattivi. non farli tramortire, ufando di tratto in tratto un profumo: qualche profumo di legni odoriferi o di carni arroftite per mantenerli vegeti e fani.

\section{A P O I I I.}

Del mantenerli, o fia palcerli.

Sccome dalla buona o cattiva qualità de' cibi dipen. $\checkmark$ de la buona o cattiva fanità dé Corpi, così devefi ufar diligenza a mantenere i Cavalleri con foglia proporzionata al lor naturale. E perchè tra Morarial. cuni fono di pola, cioè di prima buttata, altri di broo. za, cioè di più butate, alcuni cafalivi o di terten morbido, ed altri di terren magro, effendo neceffario fervirfi di tutti, fi ufs quefta cautela. Dal principio firs. chè foro avanzati delle quattro, fi cibino di quai Mo- della forati $f_{i}$ vogliono cominciando peró dalli più giovani, ec. glia. cettuato il primo pafto dopo ciafcuna dormita, quale deve effer di foglia di bronza. In fine poi, ciò nelli ultimi tre o quattro giorni $f_{1}$ cibino fempre di quelli di bronza e nagri, perchè la foglia di pola o morbida per la fua interna umidità lor nuoce, e vanno pofcia a male fopra $i$ bofchi. Nel principio li fi diano tre táde papafti firfi al giorno; nel progreffo pure tre pafti me-fit. diocri, feconcio fi olferverà che la mangiano; e nelli ultimi tre o quattro giorni anche quattro paft $i$ abbondanti, perchè quando abbiano ben mangia to in queft' ultimo tempo, fanno miglio:i galette. Nel principio è fine delle dormite li fi diano folamente quactro foglie fparfe una volta o due al giorno, acciocchè li primi a le. vare e gli ultimi a dormire abbiano con che nutrirfi. In cafo di continue pioggie maffime quando fono delle quattro, fi tenga pelata la foglia un giorno per l'ałtro affine di poterla afciugare, ed abbiano da mangiare. 


\section{4 \\ Ofservazioni}

Non effendo a tempo di poterla pelare un giorno per l'altro, è meglio in tal cafo che perdano un qualche pafto interrotto per farla in tanto afciugare, mentre la foglia bagnata li rende infermi. Si offervi finalmen. te di feparare dalla foglia per quanto mai fi pud le More troppo mature come nocive per la lor corruzione.

\section{C $\quad \begin{array}{lllll} & \mathrm{P} & \mathrm{O} & \mathrm{I} & \mathrm{V} \text {. }\end{array}$}

\section{Del porli al lavoro, ed ordinare li bo fcbi.}

A Guifa di nave, che dopo felice corfo ful Mare pa. A tifce naufragio in Porto, così avviene alcune volte a' Cavalleri, che dopo efferfi mantenuti belli e fani fino alla meta di tanti difagi, perifcono poi fopra i bor. chi con doppio rammarico di chi li mantenne ed al. levo. S'abbia dunque quefta attenzione: Come non dormono, nè levano mai tutti d' accordo, così non fi pongano al lavoro tutti ad un tempo; $\mathrm{ma}$ in due o tre volte, fecondo apparifcono maturi; perchè fe fono gatbi, non fanno buona galetta: Se troppo maturi, Modo a non hanno lena di lavorare. Nel levarli da' tavoloni deftrezia. non vi fif frammifchino $i$ guafti, nè $f i$ empifcano troppo le mani e i vafi per non pregiudicar alla loro delicatezza. I vafi fiano larghi e piani, vorandoli fubito e con deftrezza fopra le fafcine già preparate, affinchè 2ualità non reftino in alcuna parte offefi. Tutte le fafcine fodelle fa-no buone; ma offervo, che i Cavalleri lavorano meglio rine. fopra quelle di Moraro; forfe perchè più confacenti al lor naturale, o perchè di fua natura non affettan. dofi tanto come le altre, lafciano nel loro vaeuo maggior trafpiro dell' aria. Si afpeti qualche tempo a drizzarle e componerle in bofchi fin tanto che fiano ben Forma diftefi e attaccati i Cavalleri. Ciafcun bofco deve effere
ita de di mediocre lunghezza, compolto di fole tre o quattro bofchi. fafcine al pari, con di fotto un piano di legnetti ad ogn Qualita un pronti dalle cimature delle fafcine medefime. Que. de' luogbi.fti bofchi fi formano, come ogn' uno sà, fopra folari, fenili, 
Sorra il governo de Cavalleri.

nili, e granari, o in luoghi afciutti, dove fi poffa dar e levar l'aria, ed anche portar fuoco fe occorre. Si coprano quando è freddo, di lenzuoli più netti e leggieri; e quando è caldo, vi fi pongano fopra folamente de' le. gnetti, o fpazzadore di campo, o ambrogani, o gramigna ben fecca e purgata. In cafo di tempo umido o freddo è bene per i primi due o tre giorni profumar i poofumo. bofchi con fumo odorifero, perchè quefto li fveglia $e$ li fa ferrare.

\section{A $\quad \mathrm{P} \quad \mathrm{O}$ V.}

\section{Del fcoprire $i$ bofcbi, e far la femenza:}

A Capo di quattro giorni dopo meffi al lavoro li
re, e disfar con maniera i bofchi, acciò non fi fcaldino e vadino poi malamente le galette. Indi poi ad altri quattro giorni fi raccolgano le galette, non frammif. chiando le guafte con le buone; $e$ fi ufi attenzione Difinzione delle galotte. di riporle in luoghi frefchi, e di tenerle feparate fecondo il tempo de' Cavalleri che fono ftati meffi al lavoro per regola di cavar la feta frefca o folata. E poichè convien penfare di far la femenza per l'anno fuffeguente, fi fcelgano fra le migliori delle prime fatte tante libre, quante onzie di femenza fi defiderano. Si faccia offervazione che quelle, le quali hanno la punta più acuta fono mafchi, e quelle che l'hanno ottufa, cioè meno acuta fono femmine: pofcia infilzate, fi pongano a nafcere in luogo frefco ed afciutto, riparato dall' aria. Nate che fono le poveggie, fi lafciano accoppiare fopra le filze medefime, poi a tempo opportuno in una ed anche due volte al giorno fi levano le femmine, e fi pongano fopra un telo lino netto a far la femenza in luogo lucido e temperato, gettando via li mafchj, qualora di nuovo non abbifognaffero. Io reputo meglio la. Del fap fciar la femenza ful telo fin tanto che è paffato il cal- conferdo, perchè così corre minor pericolo di nafcere. Miti- menza. 
I 6

\section{OJervazioni}

gata che fia la ftagione, fi levi la femenza deftramente dalla tela con un coltello, bagnandola prima in vino puro, acciò nafcano a fuo tempo più vigorofi; fi faccia poi ben afciugare in fito riparato dalle formiche, da ragni, eda altri animali che la danneggiano. Ben afciurta che farà nel detro modo la femenza, fi cuftodifca in qualche valo vetriato oppure in fazzoletti, o facchetti di tela netta, in Aanza ben difefa dal freddo, perchè non parifca. In fine iftimo ottima cofa il ricordare ció ch'è debito d'ogni Criftiano l'offerir una qualche porzione di galette al facro altare in fegno di graticudine, $e$ in rendimento di grazie a Dio dator d'ogni bene.

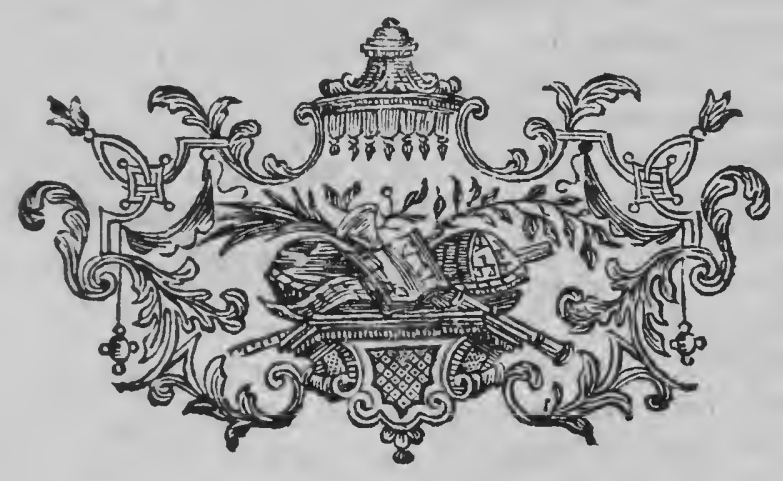




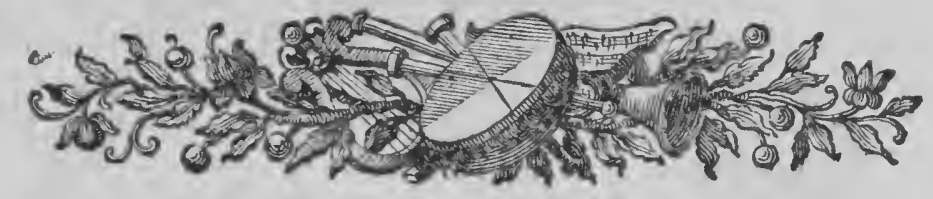 \\ OSSERVAZI ON I}

Sopra la coltivazione de' Morari.

\section{A P}

Del modo di piantar $i$ Morari.

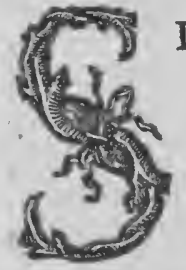

Iccome da un fodo o debole fondamento di. pende la ficurezza o la rovina d'un edifizio, cosi dalla buona o cattiva forma del piantar un' albero dipenderà la di lui buona o cattiva riufcita; particolarmente parlando dei Mo. rari da piantarfi nei terreni magri e faffofi, dove colla coltura difficilmente ancora riefcono, e fenza di efla fi perdono facilmente.

Stimo fuperfluo il dire, che le buche da piantarvi i Morari, dovercbbono prepararfi un' inverno, o un'eftate avanti, della fondezza di circa due piedi, e della larghezza di fei. Non credo però fuperfluo, ma anzi reputo neceffario il ricordare che le bine de' Morari in tali luoghi fi piantino fempre a foffa, la quale perchè non fia di troppo difpendio, $f_{i}$ formerà in quefta maniera : Si ari prima il terreno almeno per la larghezza di piedi fei; poi fi fcavi detta aratura, indi fi replichi lo fteffo, e pofcia fi ari un'altra volta bene, favando in quefta terza aratura folamente le buche diftanti l'una dall' altra tre pertiche. Piantati li Morari nella forma che $f($ dirà, fi ripone nella foffa 12 terra fcavata, rifparmiandofi in tal modo la terza parte della fpefa, e riufcendo l'impianto, come fe foffe 
tutta fcavata. Se permefio mi folfe oltrapaftare i limiti del mio affunto, fuggerirei bene il non ommettere di fcavare la folla intieramente in quelle fituazioni foggette all' umidita, nelle quali fogliono feccarfi li Morari fpezialmente in certe annate piovofe, come appunto fuccedete pochi anni fa nelle consrade di Sandrà, Colà, Gaftelnuovo e fimili, dilatando la medefima folla, e profondandola un terzo di più dell ordinario, per formarvi nel fondo alla rinfufa un piano di fafl, che fervono a prefervargli dall' umido, e rendergli men fottopolti a tale pericolo.

I Morari fi poffono piantare in due Stagioni, cioè in quarantia di San Martino, oppure in Quarefima, in tempo però che la terra fía afciutta. Quefti fiano de Mopa- di calma, perchè in tali terreni troppo foggetti all' ariri.

dità i falvatici fe s'incalmano quando fono piantati, di rado riefcono. Siano parimente di buona forte, e di mediocre altezza, piantandoli fondi poco meno di due piedi, con riguardo, che quei che foffero curvi, reftino rivolti inverfo a mezzo dì, affin che fi raddriz-

Forma zino. Nel piantarli fi empifcano le buche di rerra di pian- fenza ghiara o faffi, fupplendo con altra terra di rive, - cavedagne, frapponendovi ancora delle glebe di codego in mancanza di letame. Subito piantati, fi cuoprino i tagli di fterco bovino, mantenendoli così co. perti finchè germoglino, cioè buttino, onde non penetri l'acqua, o il rigore del freddo. Si veftano poi di canne per riparargli dagli ardori del fole, ed in alcuni fiti s'armino ancora di fpine contro gl' infulti degli animali mantenendoli in sì fatta guifa almeno cinque anni.

$$
\text { C A } \quad \mathrm{P} \quad \mathrm{O} \text { I I. }
$$

\section{Dell allevare e potare i Morari.}

TOn creda chiunque brama di allevar Morari, $\mathrm{d}^{\mathrm{s}}$. 1 aver fatto quanto bafta piantandoli nella fuddetia forma, perche febbene quefto fia il fondamen= 
Sopra la coltivazione de' Morari.

to, farà fempre il meno rifpetto a tutta la fabbrica. Ciò che più importa, fi è l'allevarli anche in modo debico, acciò d'anno in anno $f_{1}$ avanzino fempre più, e $\mathrm{fi}$ confervino.

Non rincrefca dunque al Padrone di vifitare alle volte quefte piante, principalmente quando fon picciole, e fubito che han germogliato, farle fchiarire ne' germoglj o fiano butti, acciocchè divengano più forti quei che vi reftano. Quando poi $\mathrm{i}$ detti butti abbiano fatto il legno, che fuol effere in Maggio, fi ri. ducano a foli tre per pianta, e a due nelle piante più debili; non mai a quattro, perchè col tempo non potendo fcorrer le pioggie per la troppa unione de' rami corrodono il tronco, e $s$ ' infinuano nelle piante medefrme. Ridotti che frano $i$ Morari in tal guifa, nel terzo anno, ed anche nel fecondo fe hanno forza, fi facciano teftare in Quarefima a tempo opportuno i fuddetti due o tre butti, che formano i primi rami ful tronco, alti un palmo poco più poco meno fecondo la qualità della pianta e del terreno, ma fem. pre appreffo l'occhio. Colla medefima regola del pri. mo anno s'allevano anco fopra cadauno di quefti primi rami foli altri due o tre germoglj, i quali doveranno formare i fecondi rami. Similmente a capo di due altri anni fi teftano li detti fecondi rami prima che butrino, e s'allevano pure nello fteffo modo non più di tre butti per cadauno de' medefimi rami. In tutio quefto tempo non è utile valerfi della foglia perchè reftino più vigorofi.

Pafiati li fuddetti quattro o cinque anni, deve il $\mathrm{Pa}$. drone allevarli piuttofto baffi, acciò col tempo ombreg. Del pogino il pedone; e farli potare ogni due anni fin che s'arli. accorne che ceffano di crefcere con egual vigore, facen. dogli lafciare due o tre foli occhi per rano, col taglio tondo e vicino all' occhio, e levar i rami fuperfiai. Non refti di farli fchiarire anco dopo cadauna potatura dalla fuperfluità dei germoglj fino al decimo an. no. Venuto poi il tempo in cui crefcono con minor B 2 vigo- 
20

\section{Ofervazioni}

vigore, deve farli potare folamente ogni tre anni nella ftefra maniera. Fatti alquanto vecchj, comecchè hanno ancor meno di vigore, farà meglio potarti folo o. gni quattro anni, laf́ciandovi un' occhio o due per ramo, ed alcuni anche a groppo, cioè al fegno della po. tatura antecedente. Quelli finalmente che decadono, $f_{1}$ teftino avanti che germoglino, alli primi, fecondi, - terzi groppi fopra il tronco fecondo il lor vigore, coprendo a tagli di Aterco bovino, e fi allevino nel mo. do detro di fopra come fe foffero giovani.

E quì non poffo mancare di raccomandar ai Padro. ni con tutta premura che facciano fcelta d' Uomini prattici ed onefi, procurando che fiano fempre i medefimi a far le potature. Nè anche polto lorare quell" ufo peffrmo in alcuni luoghi introdotto $d$ 'intereflare gli

Delle Operaj nella legna. Si piglino dunque a giornata, o al perfone e seinpo di posarli. più ad'un tanto al piede, con l'obbligo di far le fafcine di giorno in giorno, dal che ne ridonda un fommo vantaggio. Voglio per ultimo ricordare ciò, che a tutti è già noto, ma importa affai, di non differire a po. tarli più di una fettimana all' altra dopo pelati, e mai pallata la metà di Giugno, eflendo allora troppo a. varizata la Atagione.

\section{A P O I I I.}

\section{Del coltivar la terra intorno a' Morari.}

CUggerito il modo di allevare i Morari, rimane ora $\omega$ veder la maniera di coltivare la terra, dove fono pisntati. Infiftano adunque i Padroni a farla coltiva. re a debiti tempi, poichè da ciò dipende in gran parte la buona riufcita di tali piante; loschè ca' Villici d' ordinario trafcurafi facilmente.

Tre volte all' anno devon' effere ben arati, e zappati nel feguente modo: La prima volta fi diftolgono con due folchi per parte, e fi zappano a San Martino, e poi fi colano cioè fe gli dà la terra avanti che 


\section{Sopra la coltivazione de' Morari:}

s'agghiacci, con detti due folchi. La feconda volta fimilmente fi diftolgono, e zappano a Pafqua, col fol- Quanticare anche la vanezza che fia almeno di tre folchi, tano nelungo alle cavedagne, $e$ in fine di Maggio fi colano, cefavio a formando in' oltre la detta vanezza. La terza volta parimente fi diftolgono, e fi zappano nel principio di Luglio, folcando di nuovo la fuddetta vanezza, e nel fine di detto mefe o nel principio d'Agofto fi collano, riformando la vanezza predetta. Quelli poi che fono piantati ne' prati, o né pafcoli, fi coltivano con una vanezza per parte oltre $i$ due folchi. E ficcome quefti in tali fiti fogliono patire il caldo più degli altri, qualor $s^{\prime}$ accorga che per queft effetto decadono, ruggerirei a fcavarvi un foffo per parte nel codego laterale alla detta vanezza, otturandolo di mano in mano che fi fcava, a ffinchè fi rimettino. A quelli finalmerre fituati nel mezzo delli campi ara. tivi, offervata la segola di fopra, bafteranno i foli cue folchi d'ambe le parti, guardandoli tutti dall' of. fenderli coll' aratro.

\section{A $\mathrm{P}$ O $\mathrm{I}$ V.}

\section{Del modo d'ingrafjare i Morari.}

DEr ingraffare i Morari è buono ogni letame, maffime fe fia confumato. Ma come quefti fon luoghi, dov' è fcarfezza di graffa, e i Villici di mal' animo foffrono d'effere taflatti in quefto particolare, e dall altra parte fendo pur troppo neceffario l' impin. guargli; perciò fénza difguftar i primi, fi potrà beneficar i fecondi, medicandoli fe non altro, come fuol dir$f i$, del loro medefimo pelo. Di grazia lor non $f_{i}$ faccia quefto torto, $f_{i}$ riferbino $i$ letti dei Cavalleri, $e$ fe ve ne è anche la Gallinella o fra fterco di Galline; e con quefti $s^{\prime}$ ingraffino almeno i più giovani, e bifognofi; fpecialmente qualora fono di potatura; tra quali crederei li fituati lateralmente alle Siepi, come più fog-

$$
\text { B } 3 \text { getti }
$$


getti a patire; oppure $\mathrm{fi}$ benefichino di menadize, o di Noncal- codeghi. Non lodo il calzarli di terra, come fanno zerra. alcuni, perchè reftando in quella parte ammollita la fcorza, o radicando in alto, fi rendono più efpofti all' intemperie delle ftagioni.

\section{A P $\mathrm{O} \quad \mathrm{V}$.}

\section{Del. trapiantarli.}

TTRa i molti privilegi che gode l'agricoltura fopra 1 tutte le altre profeffioni, non è di si poco tilievo quefto; che mutando talora l'ingegnofo agricoltore 1 . idea, e riflolvendo riordinare Arbori già piantati, lo fa direi quafi con quella facilita, con cui un ottimo Capitano mette in fquadra, e riordina il fuo Efercito. Cofa invidiabile a chi dopo aver fabbricato un palagio, non può rimediare agli errori, e foddisfare alle nuove idee fenza prima diftruggerlo.

Volendo adunque trapiantar Morari, o perchè fiano in luogo ove rendano incomodo, o per renderli più ordinati, purchè la groffezza del pedone non fia troppo eccedente, $f_{i}$ trapiantino in Marzo con più radici che Ia poffibile. Quefti tofto cavati fi teftano a' primi gropofjeroa- pi fopra il tronco, e fi piantano di fubito con tutta dizione do' ligenza alquanto più fondi, e ne' medefimi venti, ne' quali ritrovanfi polti prima di cavarli, come l'efperien. za chiaramente ce lo dimoftra. Si allevano poi, e fi coltivano colle medefime regole dette di fopra.

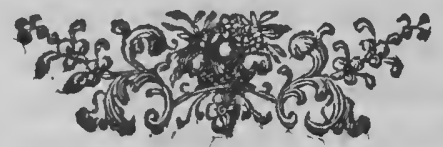




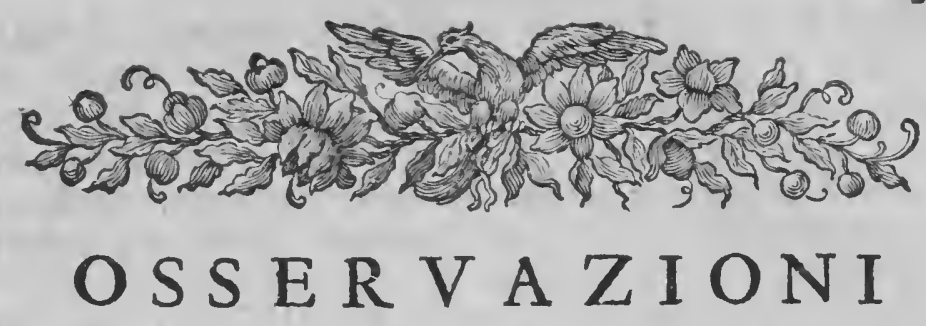

Intorno alla coltivazione delle Viti.

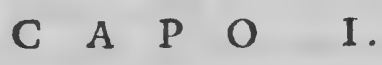

Dell impianto delle Viti, e loro foftegni.

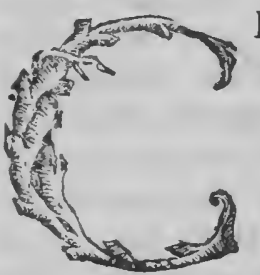

He io voglia dar regole per piantare, e coltivare le Viti, fembrerà forfe inutile cofa e fuperflua, quando effendovene tanta copia per fino nelle Città, fi può credere che ogni benchè femplice ed inefperta períuna ne abbia una fufficiente cognizione. Oftervo però talvolta fuccedere, che meno di attenzione fi ufa nelle quotidiane azioni, che nell' eftraordnarie; onde ne avviene che ancora fi manca di certe circoftanze, per trafcuraggine delle quali di minor profitto riefce ogni operazione. Chiunque perciò defidera fecondare di Uve le proprie Campagne, non ifdegni riffettere alle feguenti offervazioni.

Prima d'ogni altra cofa fi feelgano i taglıoli di buo. a forte, o nell' inteffo luogo, o ne' vicini; perchè molte volte accade che mutando qualità di terreno, mutano anche l'Uve la lor natura. Nelle Campagne magre, per le quali s'jntende dar qualche regola, li prend no quelli di Vite Cremonefe, Corvina, e Rofferta; e ne' fiti meno magri ancora di Cagnetta, Tirodola, e Vernazza, come più fertili ed ubertofe in quefti terreni. S' Qualita, averta che quella Corvina tirata in alto fenza mai po. 'quantisa tarla, produce l. Uva per vino labrufco. Siano i taglio- $t i$. B 4 


\section{Ofervazioni}

fi piuttofto curti e groffi, quali rubito tagliati, fi pone in acqua la parte che va fotterrata, evi fi lafcia per alcuni giorni, poi fe ne piantano in quarantia di fan Martino quattro per parte al pontezo, fondi almeno un piede diftefi due a due ordinatamente, e diftanti quafi un piede dalli pontezi, per così rimettere gli uni e gli altri facilmente.

Parlando de' pontezi, pare che l' Oppio fia alle Viti molto opportuno; tuttavia vedo 'dall' efperienza che nel terren magro non crefce a fufficienza per mantenerle. Cosi non lodo i Marafcari, Brognari, Olmi Rizzi e fimili, che crefcono bensì con preftezza; ma colla moltitudine de' germogli infettano i Campi : nè meno la Nogara come nociva alle Viti. Sarà perciò mede' Ponte glio piantarvi Cirezari, Olmi Bianchi, e Fraffrus; e ne' si.

firi migliori anche l'Oppio. Mi fembra cola affai utile il piantarvi ad ogni due pofte pure delli Moraretti falvatici, facili ad allevarfi negli Orti con la femenza di Moraro all' ufo Mantovano per ricavarne oltre i pali anche la foglia, allevandoli però a modo di pontezi, e non di Morari. Siano turti quefti Pontezi di mediocre grandezza, e di quelli non puramente feminati, ma tra. piantati, ponendoli fondi un piede e mezzo, all' iftefra fondezza fcavando tutto il fofro. Quefti ancora $f i$ benefichino con terra di cavedagne più che fia polfibile prima d' otturar il foffo intieramente.

\section{A $\quad \boldsymbol{P}$ O II.}

\section{Dell allevare le Viti, e i Pontezi.}

T E Viti fi fogliono teftare tre o quatro anni dopo nova. Nel primo anno fi allevano da detti occhj due zami per vite, liberi dalla fuperfluita de germogli; la qual fuperfluità $f_{i}$ doverà levare dal tronco delle Viti in ogni tempo. Nel fecondo anno pure in Quarefima fi rio. duco. 
ducono i detti rami ad un folo per vite il più forte col teftare anche quefto, lafciandovi due foli occhj. Nel terzo anno poi fi potano parimente in Quarefima, con Dolpotare lafciarvi un folo ramo per Vite fatto a tirella il più vigorofo con due occhj fra la ligatura del medefimo ramo e la teftatura della Vite; e così fuffeguentemente, affinchè producano i rami per l'anno feguente da formar le folite tirelle ad un folo ramo per vite il principale, recidendo i rimanenti: alzandole d' anno in anno fin che fono arrivate alla teftatura delli rami dell. arbore. In quefti anni fi tengono foftenute alle frofche e pali per non aggravare il pontezo non per anco ba. ftante a foftenerle. Ridotte che fiano alla fuddetta teftatura dell arbore per quanto mai fia polfibile fi mantenghino a tal fegno, potandole fempre in luna vecchia nell'ifteffo nindo. Siccome col progreffo del tempo taluna trafcorre l'antedetta teftatura, così in tal cafo s'abbia attenzione di lafciarvi fotto la legatura un germoglio detto da' Villici fnerone con due occhj, e non effendovi di teftar la Vite al groppo che pare opportuno, acciò produca i confueri due rami, e non oltre. paffi il fegno predetto Qualos occorra in qualunque età rimetterle, fi trattorano bella feguente maniera: $\mathbf{S i}$ fradica la metà d'una delle viti dalla parte più nume. rofa, e fi fotterra ben fonda paffando a quella mancan- dol tratte con $\mathrm{i}$ due rami, che fi troverà avere, difpofti e re-torarle. cifi a guifa dei taglioli, da'quali fi produrranno in breve tempo due viti. In cafo po: che detti rami non forfero fufficienti ed opportuni, è neceffario teftarli ambidue, ed allevarvi tanti rami da trattorare l'anno fucceffivo, quante Viti fi defiderano rimettere

Tutti gli arbori che fervnno di foftegno alle Viti fi teftano dal pedone preffo l'occhio in Quarefima tre o quattro anni dopo piantati, alti due piedi circa $\mathrm{Nel}$ primo anno ful fine d. Aprile fi allevano da quefti quattro ordinati raıni di moderata larghezza, acciò fiano dominate dal Sole le Viti, e divenut gagliardi poffino foftener le tirelle, mantenendoli netti dai butti fuperflui. 


\section{6

flui. Paffati altri tre anni, fi teftano parimente in Qua: refima li detti quattro rami appreffo all'occhio alti pu. re due piedi circa dal tronco. Per cadauno di detti ra. Dell' alle mi s'allevino due o tre pali, tagliandone dalla ftefre vare ipa teftatura una terza parte all' anno, acciò reflino an. nualmente provvedute le Viti In detto corfo d' anni non fi debbono pelare, teftando i pali fucceffivamente fempre nel medefima fito.

\section{A $\quad \mathrm{P} \quad \mathrm{O}$ I I I.}

Del coltivare la terra intorno alle Viti.

A Ncora le viti fi arano tre volte all' anno, diftolenA dole, zappandole, e collandole ogni volra con due folchi per parte, cioè a San Martino, in Primavera, e in Luglio, fin che fono ridotte allo Rato di compiuta tirella. Dopo quefto tempo fi coítivano nel feguente modo: Si diftolgono in Quarefima pure con due folchi per parte, e fi zappano, fi collano poi con un folco per parte, allorchè principiano a germogliare, e con l'altro folco folamente in Maggio, acciò vengano me. no infeftate dall' erba. Di nuovo fi devono diftorte almeno avanti San Giacomo di Luglio con un folco per parte, ed in Norembre collare col detto folco. I VilDella di- lici vi ftiano diftanti colli feminati tre piedi per parte Aanzacon per poterle coltivare nel detto modo. Così pure non lifernina- vi lafcino dopo la vindemmia andare gli animali a traverfo, dal che ne viene la rovina delle Viti medefime.

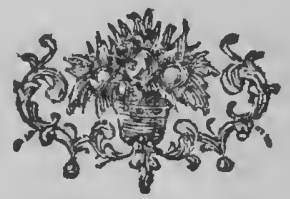




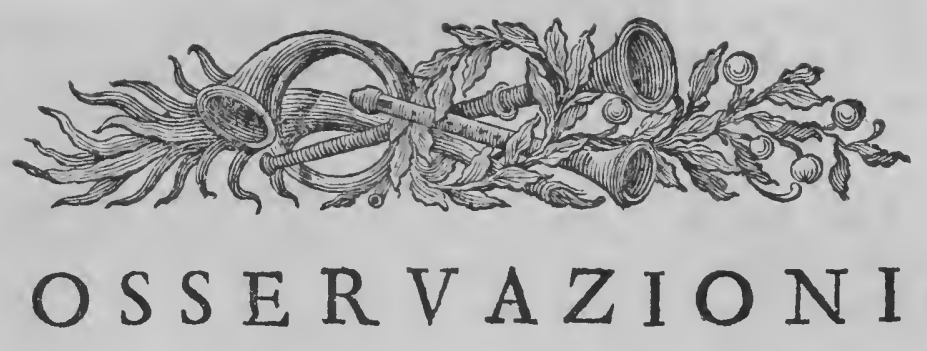

Sopra la Coltivazione delle Siepi.

\section{A P O I.}

Impianto delle Siepi.

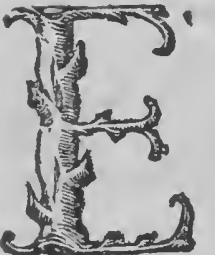

Così fearfo a' giorni noftri il Territorio Verunefe di legne, almeno in quefte con. trade, che con iftupore ho udito negli anni fcorfi esfere ftate pagate nel tempo dei Cavalleri le îfcine di rovero anche ordinarie fino lire trenta per carro. E quefto non da altro procede a mio credere, che da i fvegri fatti in ogni parte, per i quali fono ftati efterminati i bofchi, e cefpugli. Offervo ancora che nelle magre Campagne vi è tanta penuria di frofche e di pali, onde foltentare le Viti, che i Padroni fono in neceffità di fpendere non poco danaro per farne provvigione. Be. sedetro dunque fia fempre chi invento la maniera di renderle fertili di legne e di pali, coll' introdurre le Sie. pi fenza perdere l'entrate de i fvegri. Voleffe Iddio, che ogni Padrone s'innamoraffe d'una tal coltivazione, che la noltra Patria coftretta tanto non farebbe a ricorrere a'bofchi forentieri con non poca perdita di foldo. Nè creda qui alcuno, che fi richiedane molti anni a formarle, mentre in brevifimo tempo, come dall efperienza fi vede, fil allevano, e tagliano.

Molte fono le piante, atte a formar dette Siepi: Ma fopra 
fopra tutte il Bolcefrino ne" luoghi magri e faffof rie. Qrealitis fee a merariglia, legno che da foltegno e da fuoco non pi. Sic la cede a qualunque alcro più forte. Quefti frano di que' trapiantati, e in quarantia di S.Martino, o in Marzo fi piantino fondi un piede e mezzo, ed altrettanto diftanti l'un dall' altro, a folfo largo almeno due pie. di, beneficandoli maffme nel magro con terra di care. dagne prima di fotterrarli. Il Períco ancora è ottimo, piantato nella Atefia forma che-il Bolcefrino, purche il terreno non fia così magro. Crederei opportuna anche la Nogara, piantata nell' ifteffo modo, ma in maggior diftanza del Bolcefrino, mentre viene in ogni luogo. Di non poco utile finalmente faran quelle formate di Moraro, ed è facile l'introdurle, o colle radici levate dalle Morarie piantate a foffo; o colla femenza degli fteffi Morari feminata in quarantia di San Mastino a folco in terren ben lavorato, mefcolata peru con acqua, $e$ ferco bovino, non fotterrandola troppo. Nati poi li Moraretti fi nettano dall erba, e fi riducono in una fol fila diftanti un piede l'm dall' altro, fotterrando affatto il detto folco.

Per non occupar il terreno tali Siepi fi piantano a i lati della terra meno fruttifera, cioè lungo a i prati, a gli arzeri, a i palcoli, a i folfi, e dietro alle marogne, e ne' confini de' campi. Saria perciò di grande vantaggio a Padroni l'allevarfi negli Orti ogni forta di piante, e fpecialmente di Moraro, come dote neceflaria, e principale di quefte noftre Campagne.

\section{A P C II.}

\section{Modo di allevare le Siepi:}

QI allevano dette Siepi col teftarle a capo di trē a $\checkmark$ quattro anni al più, alquanto prima che cominciano a buttare, baffe 2 terra, cioè agli ultimi occhj. Dopo teftate convien, quando hanno germogliato, fchiazire i germogli accio divengan più forfi quei che vi re: ftano, 
ftano, ed allorchè averanno fatto il legno ridurne quat: tro, circa per pianta, di qué più dritti, per poterle a. rare liberamente fatti grandi. Chi poi bramafie unicamente la legna per fafcine, e non per pali, tralafci di fchiarirle. Paffati altri tre o quattro anni, fecondo la qualità del terreno, $f \mathrm{f}$ a novamente il taglio in quarantia di S. Martino, o in Marzo, pure baffo alla te. ftatura prima, e così fucceffivamente. Si guardino que. fte Siepi, come anche ogn' altra pianta, dagli Animali, maffime quando fon di prima buttata.

\section{A P O I I I.}

Del coltivare la terra intorno alle Siepi. dette volgarmente Ceje.

T E Siepi fi devono arare tre volte all' anuo con due folchi per parte, ciò̀ diftorle, zapparle, e col. larle in Marzo, in Luglio, e in Novembre, e non po. tendole arar da una parte, fi facciano vangare.

$S$ 'averte in ultimo luogo di maj non permettere che vengano arate, o vangate Piante di qual fi fia forta, qualor il terreno fia troppo bagnato, o troppo a. fciutso; così pure che non fiano pelate fe non paffato il mefe di Agono, e li Morari fe non brumati; incari. cando perciò i Lavorenti a feminarfi il bifognevole di paftura affinchè non fiano neceffitati a pelarle prima di detto tempo. Il tutto però riefcirà vano quando non fi metta per fondamento di tali diligenze, fpefe e fatiche il fanto timor di Dio.

\section{$\begin{array}{llll}F & I & N & E\end{array}$}

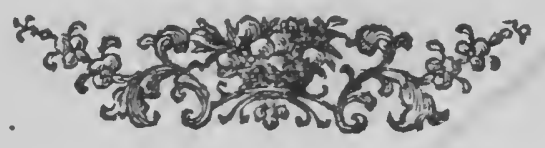




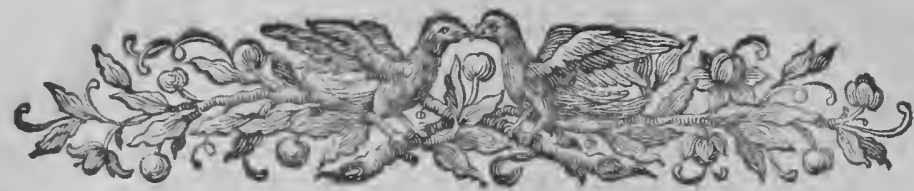

$\mathrm{T}$
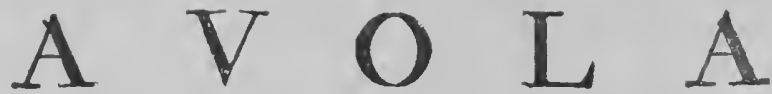

Delle cose piu offervabili.

\section{Sopra il governo de' Cavalleri.}

Cap. I. El tempoe modo di mettere i Cavalluri. Del mettere e covar la Semenza. Qualità delle ftanze.

Cap. II. Del modo di allevarli.

pag. 9 . ivi.

IO. ivi .

Separazione fecondo l'eià. Modo di mutarli.

USo Trentino nel mutarli.

Ulo del fuoco.

Regola del froco.

Ufo del termometro.

Altra Separazione.

Del fumo, ftrepiti, odori cattivi e profumo.

Cap. III. Del mantenerli o fia pafcerli.

Qualitì della foglia.

Quantità de' pafti.

Cap. IV. Dil porli al lavoro, ed ordinare li bofcbi.

Modo e defrezza in governarli.

Oualità delle fascine.

Formalità de' bofcbi.

Or alità de' luogbi.

Profumo.

Cap. V: Dol Scoprire $i$ borcbi, e far la Semenza.

Diftinzione delle gallette.

Dil fare e conservar la Jemenza:
I I.

ivi .

ivi.

I2.

ivi.

ivi.

ivi .

I3.

ivi.

ivi:

ivi .

I4.

ivi.

ivi.

ivi.

I 5 . ivi.

ivi.

ivi.

ivi. 


\section{Sopra la coltivazione de' Morari.}

Cap. I. El modo di piantar $i$ Morari. Qualitì de' Morari. Forma di piantarli.

Cap. II De!l' allevare e poiare $i$ Morari. Del teftarli.

Del potarli.

Delle perfone e tzmpo di potarli.

Cap. III. Del coltivar la terra intorno a' Morari.

Quantità del terreno neceffario a coltivarli.

Cap. IV. Del modo d'ingrafsare i Morari.

Non calzarli di terra.

pag: I7.

IX. ivi: ivi.

I9.

ivi.

20.

ivi .

2 I.

ivi.

22.

ivi.

ivi .

\section{Intorno alla coltivazione delle Viti.}

Cap. I. DEll' impianto delle Viti, e loro foftegni. 1 Qualità e quantità de' taglioli. Qualità de' Pontezi.

Cap. II. Dell' allevare le Viti, e i Pontezi.

Del potare le Viti.

Del trattorarle.

Dell' allevare i pali.

Cap. III. Del coltivare la terra intorno alle Viti, Della diftanza con li feminati.

23. ivi.

24, ivi".

25. ivi . 26. ivi . ivi .

\section{Sopra la coltivazione delle Siepi.}

Cap. I. T Mpianto delle Siepi

27:

28.

Cap. II. Modo di allevare le Siepi.

Cap. III. Del coltivare la terra intorno alle Siepi. ivi:

29.

\section{FINE DELLA TAVOLA.}




\section{O S S E R VAZ I O N I SOPRA LA CULTURA}

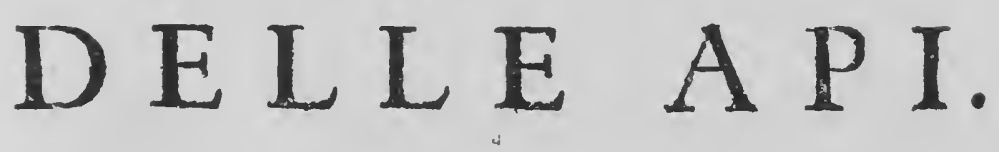





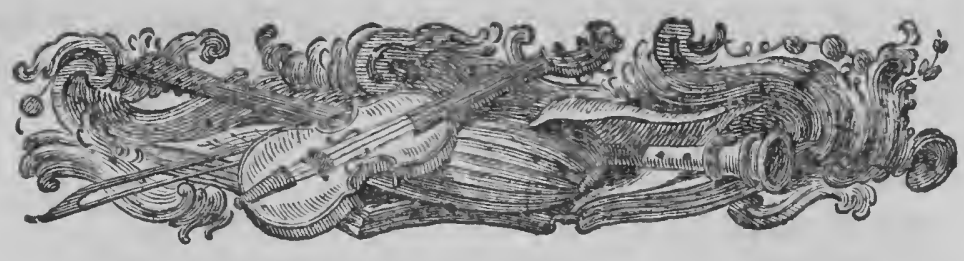

\section{O S S E R V A Z I O N I}

Sopra la cultura delle Api .

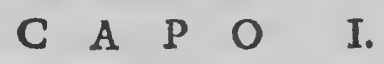

Della qualità delle Api, Re, e Peccbioni.

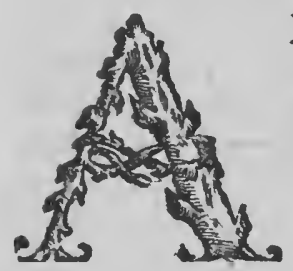

Bbiamo da Ariftotile nel fuo Libro de longa Vita cortiffimo effere il vivere degl' Infetti, e pel contrario affai più lungo d'alcuni altri Animali quello delle A pi, per la ragione che fupplifcono que. fte col miele, cibo molto falutevole, di cui continuamente fi pafcono, a quell' u. mido, e calido, che loro manca, fino agli anni fei vivend o, come egli fcrive nel 5. della Storia degli Animal, benchè in Virgilio fi abbia fino alli fette, e nel Spettacolo della Natura fino alli otto, e di vantaggio ancora. Perilchè oltre l'altre molte prerogative, anchè per quefta meritano effere tenute in maggior pregio di quello che in fatti quì fi tengono; non dovendofi però far di tutte la medefima fima, mentre non fono tutte ugualmente fruttifere, per effervene di molta forte.

Si trovano delle Api grandi, nere, e pelofe: altre men grandi, ma più rotonde, fofche, e di pelo rollo: altre più picciole, ma lunghe, di color alquanto più chiaro: altre in fine ancor pit picciole, minute, più lunghe, più agili al volo, lifcie, di ventre acuto, e di cobot d'oro; equelte fono appunto le più fruttifere, come A 2 di. 
dice l'iftefro Ariftotile, delle quali conviene averne più cura delle altre, e lo approva anche Virgilio nel primo della Georgica

Apibus quanta experientia parcis.

- come altri leggono, parvis

e nel 4. della Georgica:

Ardentes auro, of paribus lita corpora guttis:

Una d'effe fe ne trova per ogni fciame più lunga; e più grofla di tutte fenza aculeo, o fia pungiglione, di color più vivo,e più rofficcio, di portamento grave, e polato, che tengono in conto di loro Madre, e $\mathrm{Re}$. gina, come unica atta alla generazione, e fempre feconda; effendo le reftanti Vergini e con l'aculeo; la quale, corteggiata continuamente da dieci, o dodeci Api, va deponendo le fue Ova in diverfe cellette, ed efce rade volte dall' Alveare, come narra il Pivati nel fuo Dizionario fcientifico, abenchè nel Spettacolo della $\mathrm{Na}$ tura Tomo I. Dialogo 6. f tenga per certo, che abbia anch effa il pungiglione, e poffi effere corteggiata da molti Pecchioni deftinatile, come fi crede, per la generazione; e ch'effa polfa produrre per fino a fei, o fet. se mila figliuoli all'anno.

Tutte pero concorrono (fegue il detto Autore) alla di effa fecondità, rifcaldando alcune di dette Ova finchè diventano vermiccivoli : altre innafiandoli, ed altre provvedendoli di nutrimento, finchè fi fieno a poco a poco ingroffati: altre conducendoli feco a fucchiare $i$ fiori, riguardatida ogn' una come proprj figlj; e nel fine dell" Eftate, in cui finifce la generazione, uccidono tutti li mafchi, cioè i Pecchioni fupertlui :

Ignavum fucos pecus a prasepibus arcent.

Virgilio in detto lib. 4. perchè quefti, come ferive il Columella nel fuo libro 9 , de'Re ruftica, non folo race colgono, ma anzi confumano il raccolto altrui : nam neque alimenta congerit, 6 ab aliis invezt a confunit; reggendo, e governando mirabilmente il tutro forco una fola Regina, o, come altri vogliono, fotto un folo $\mathrm{Re}$.

IiRe fono lifci, fenza pelo, fenza pungiglione, e più 
grandi delle Api ordinarie, dal detto Virgilio diftinti in due forti nel medefimo lib. 4., cioè; altri fquallidi, di color cenericcio, e pigri ; quali fono i peggiori; altri rifplendenti a guifa dell' oro, e di bell' afpetto, ed agili : equefti fono i migliori:

Altera erit maculis arro yualentibus ardens:

(Nam dio funt genera) bic melior, infignis, oo ore.

Et rutilis clarus Squamis : ille borridus alter.

Dalle memorie dell Accademia delle Scienze rapportate nel Spettacolo della Natura abbiamo, che i Pecchioni fono fenza pungiglione, e di un color più fcuro delle Api; e di quefti ve ne fonodi due forti; alcuni più lunghi, e più groff delle Api; altri della grandezza delle A pi ordinarie, quali fi crede, ch' effe fi rifervino pe' bifogni dell' anno avvenire; ciò argomentandofi dal vedere la Regina nella Primavera nuovamente feconda.

\section{A P $\quad$ O I I.}

Dell'opporatusan Situazione, e pafusea por le Api.

CI proccuri di callocar len - foggetti troppo al vento, perchè impedirebbe loro 1"ulcita alla Campagna, e maggiormente il ritorno che fanno cariche di cibo; così Virgilio lib. 4.

Quo neque fit ventis aditus (nam pabula venti

Ferre domum probibent)

nè foggetti molto all' Eco, perchè il rimbombo di que. fta le farebbe fuggire, come offerva Lucrezio lib. 4 . de rerum Natura.

1 Giardini, Orti; o altri luoghi all'aperto, ma chiu$f$, onde non poffino effere difturbate, perchè amantidel. la quiete, e non efpofti nè al troppo freddo, nè al troppo caldo, fono i più opportuni; lontani però dalle Tegi, e dalli $\mathrm{Naff}$, perchè quefte piante a detta del Columella nel citato libro 9. fono loro nocive: At tili a fola ex omni. bus funt nocentes; taxi repudiantur.

Gli Alveari, o fieno Caffette delle Api, fi debbono porre 
porre preffo qualche muraglia, alte da terra circa tre piedi, perchè non fieno sł facilmente moleftate dalle $\mathrm{Lu}$. certole, Vermi, Bifcie, o altri Animali nocivi, ne' da' Venti: ed, in quanto vi foffe timore de' ladri, fi debbono tenere fotto l'occhio della Cafa del Padrone, o del $\mathrm{Cu}$ ftode, come infegna il citaro Columella lib. 9.

Quefte Caffete debbono effere foftenute da pietre, o fodi legni, e che abbiano qualche coperto di fopra per di. fefa dalla intemperie delle ftagioni; avvertendo che fie. no rivolte a mezzo giorno, partecipando alquanto di Le. vante, perchè le A pi poffino fortire alla paltura nel pri. mo fpuntare del Sole; ut apricum, il detto Columella, babeant Apes maiutinum egrefsum; e, fe foffero molte, fi mettano pure flatamente unite, che ciò riefce anzi di maggior vantaggio, perchè, effendo Animali focia. bili, quanto più fono vicine, tanto più a vicenda lavorano.

In oltre è neceffario che le dette Cafsette fieno difefe dall' ombra di qualche pianta, o altro dal Sole dell E. ftate, acciocchè il troppo caldo non difecti; ae liquefaccia il miele; in modo però che non refti impediro al. le Api il libero tranfito, c ann imbarazzi gli Alveari: Obviaque bospitiis teneat frondentibus Arbos. Lo ftefso Virgilio nel citato Lib. 4. della Georgica.

I Luoghi circonvicini, oltre quella quantità de' pra. ti, e fruttari, che parerà propria fecondo la moltitudine delle A pi, fi rendano fertili di erbe, e di fori, come fo. no il Timo, la Melifsa, Ifopo, Boragine, Maggioraua, Salvia, Rofmerino, Lavanda, Puleggio, Spigo, Giglj, Viole, Rofe, Papaveri, Trifoglio, ed altre di qual fi fia forte confacential terreno, perchè, al dir del detto $\mathrm{Lu}$. crezio, leA pi fucchiano il loro pafcolo da qualunque fiore:

Floriferis in Saltibus omnia libant.

e particolarmente in quefti paefi le prime quartro, come più florifere dell' altre; avvertendo che di quefte, o di quelle de' prati, ovvero de' campi ve ne fieno fem. pre in abbondanza, acciocchè in ogni tempo abbiano con che nutrirf, e che non vi vadino perciò gli Animali qua. dru. 
drupedi a pácolare, onde non refti illefa detta loro pa. Atura:

$$
\text { Neque oves bedique petulci: }
$$

Floribus infultent, aut errans bucula campo

Decutiat rorem, fo furgentis adterat berbes. Virgilio ful prinripio d efso Lib. 4.

Prefso la fituazione delle Api ( quando non vi fia qualche Fonte) è duopo mantener loro da vicino in po. ca eminenza alcuni Vafi riempiti fpefso d' acqua pura con entro de' faffi, o legnetti galleggianti, acciochè le $\mathrm{A}$ pi dalla vicinanza, e comodità dell acqua fi prefervino dalle infidie delle galline, ed altri Animali che le divo. rano, e non corrano pericolo di affogarfi; tenendole perciò lontane anche dall' acque ftagnanti; ma particolar. mente dalle putride, e cosi da qualunque altro odor cattivo, quale al dir d'Ariftotile nella fua ftoria degli Animali lib. 9. fommamente abborifcono.

\section{A P $\mathrm{O}$ II I.}

Degli Alveari, o fieno Casete per le Api, tempo di provvederle, e modo di trasportarle.

Olti fono gli abufi, che apportano pregiudizio alla 1 confervazion delle A pi nella materia, e forma delle Caffete, principalmente in quefti Territorj, voglio dire, il dannofifimo coltume di porle in Alveari fabbrica. ti di legnami amari, da effe abborriti, uniti con chiodi, ed altri ferri, ovvero di fcorze d' Albero, o d'Albe. ri forati, diritti in piedi, e l'eftremità di effi co' buc. chi del tranfito loro troppo grandi con altri difordini di gravifimo danno, difperdimento delle $\mathrm{Api}$, e pregiudizio del loro lavoro; perchè , ficcome le A pi principiano i favi, cioè i fieli dall' alto, e li fabbricano a perpendicolo, come fi offerva nel fpettacolo della Natura Tom.I. Dialogo 6 ; così gl' Alveari effendo in tal guifa formati, riefce il fito tanto angufto, onde non fi poffono far quelle operazioni, delle quali a fuo luogo fi tratterà . 
Per levar adunque codefti pregiudizj, debbonfi fabbricare gl' Alveari di tavole di Pezzo, Pino, Larize, o al. tro Albero odorifero, ben fecche, lifcie, e pulite al di fuori, e nette da ogni tarlo della groffezza d' un oncia, ma rozze al di dentro, commefre con incaltri, e ftecchi ben forti fenza ferro di veruna forte, porche yuefto le fa. rebbe in qualche parte putrefare, come defcrive il Falchini ScrittorFiorentino nella fua nuova e vaga Iftruzio. ne intorno le Api, ftampata in Firenze l'anno 1747.

Riguardo poi alla forma di quefti Alveari (fegue il detto Autore) dee effer quadrilunga, a guifa appunto di Caffetta, non diritti in piedi, ma rivolti ediftefi al lungo, perchè le Api vi fi mantengono, ed albergano più Jungamente, e comodamente; della longhezza d'un braccio, ed un terzo di Firenze, che fa due piedi, e oncie tre di Verona, fecondo l' infegnamento del Perini, dell' altezza, e larghezza di mezzo braccio Fiorentino, il quale fa oncie dieci, e un quarto del piede Veronefe : con cinque buchi nel mezzo della facciata pel tranfito delle Api , della grandezza delll loro Ke, e non più : $A n$ guftos babeant aditus, parla chiaro Virgillio; perchè fieno meno moleftate dagli Animali; fatti però trafverfalmente: avvertendo, che fia ben chinfa ogn' altra fiffura con fterco bovino frefco, perchè allo fcuro lavorano meglio e nel Verno reftino più riparate dalla rigidezza dell' aere. Nell' altezza, e larghezza de' quali Alveari mi pare che il Falchini prenda qualche sbaglio; imperciocchè, formando le Api i loro favi a' perpendicolo, della lunghezza d' un piede, e larghezza di mezzo piede, come fag. giamente fi offerva nel Spettacolo della Natura; ogn' u. no vede dover effere quefte mifure parimenti di oncie dodeci, che compongono appunto il piede, fe vi hanno da capire i fieli medefimi giufta la quì anneffa figura. A. 
Sopra la cultura delle Api.

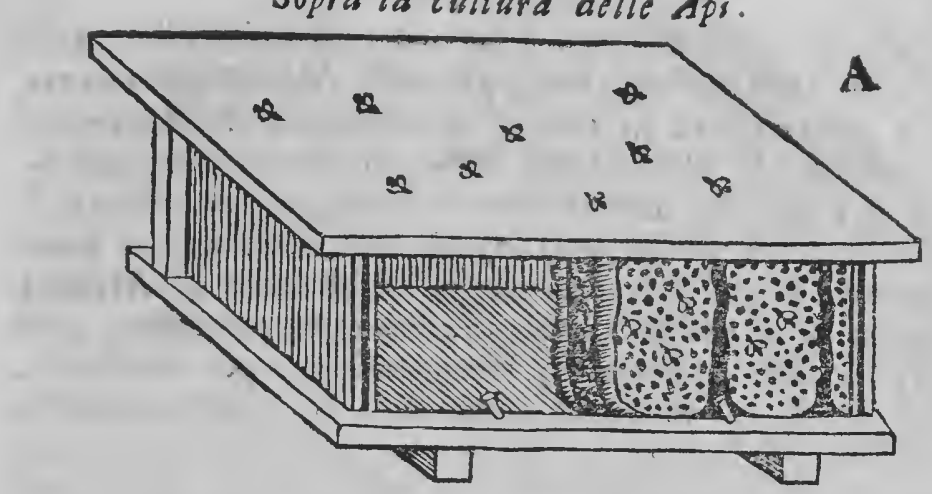

S' avverte che i lati di dette Cafsette fieno fermati in dentro dal piano almeno un' oncia, ed il coperchio in fuori tre oncie da tutte le parti, perchè non v'entri l'ac. qua; e che la facciata fia tutta d'un pezzo, e lifcia anche di dentro, e che pofsa aprirfi, come dalla quì impreffa figura ; per poter vifitar liberamente le Api quan.

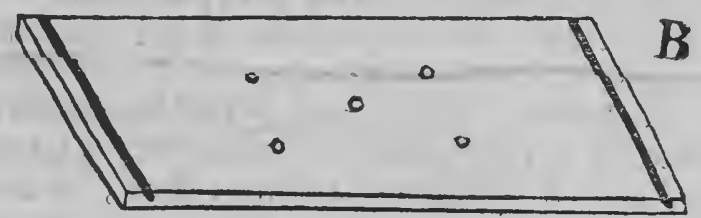

do occorra, e levar i fieli, cioè le panne, come in fe. guiro andrò defcrivendo, fenza apportar nocumento a quefti induftriofi, ed innocenti Animaletti tanto encomiati da S. Francefco di Sales, eda altri Santi: il che fi vede praticato nella Tofcana, ed in altri Stati, e Ter. ritorj fimili a quefti noftri, come fe ne fa teftimonianza l'anzidetto Falchini.

$E$, perchè non accada, come avviene a molti, di la. fciare miferamente fuggire le Api per mancanza di AI. vearj nuovi, allorchè li vecchi fono per ifciamare; convien far preparare qualche tempo innanzi le Caffette occorrentida foftituire alli detti Sciami nello ftelfo gior. no che le Api fciamano, poichè, appena levato il Sole del giorno feguente, fen volano talmente, che non è più poflibile il riaverle. 
Volendo poi trafportare da una contrada all' altra o da una Villa ad'un altra, gli detti Alveari, fi avver. te ciò doverfi fare in tempo dinotte, echetamente, coprendone gli Alveari con Pelli, o altro, per non ifmovere $\mathrm{i}$ fieli, e non irritar le Api, come offerva il Weckero lib. 8. de Secretis Apum cap. 16., da cui pure fi approva la già detta maniera di fabbricare gli Alveari medefimi con quefte parole : latitudo fit cubitalis; lon. gitudo duorum cubitorum; intendendofi, per latitudo, anche l'altezza: in confeguenza ftefi al lungo, come ho dimoftrato di fopra.

\section{C $\quad A \quad P \quad O \quad I V$.}

Della vera maniera di trattenere, e prendere le Api cbe Sciamano.

7 Utte le A pi, come offervo nel Spettacolo della Na1 tura (quando fono crefciute in tal numero, che non fi pofrono piis allowar nuovi passi fula rittrignerfi) fciamano unitamente d'accordo per abbandonare il proprio Alveare, e fuggirfene furiofamente altrove, ma non tutte, dice il Falchini, a tempi fteffi; onde conviene prima fapere, che quelle che fciamano nel mefe di Maggio, e tal volra anche in quello di Giugno, fono buone, e da praticar ogni diligenza per ripigliarle, ma quelle che fciamano dopo il detto mefe di Giugno, fono cattive, e vagabonde, per il più fenza $\mathrm{Re}$, confeguentemente dificili a trattenerfi nei loro Alveari, ancorchè fi prendano, e da non farne conto, perchè provenute da fieli creati fuori di tempo.

Due fegni offerva il Falchini precedere la loro partenza : il primo fono varj globi, che di fe fteffe formano intorno ai buchi del loro Alveare; il fecondo è un ftraordinario rumore che fanno dentro e fuori della Caffetta, in tal guifa profeguendo otto giorni circa, fin tanto ch'efcono i nuovi Re colla loro giovanile comitiva, per abbandonare ad'un tratto la loro vecchia abi. 
tazione defiderando nuova $\mathrm{Cafa}$; quella larciando fem. pre in poffeffo delle Api Vecchie, come fi raccoglie dal Spettacolo medefimo della Natura.

In quefto tempo fi rende perciò indifpenfabile l'at tenzione d'una guardia, perchè non fuggano fenza fpe. ranza di riacquiltarle, e particolarmente nelle ore più calde del giorno, nelle quali fogliono fciamare, affine di poterle trattenere nel feguente modo.

Primicramente bifogna aver pronto qualche metallo da battere più, o meno, fecondo che fono alte, o baffe, per interrompere la loro fuga, che da effe viene intraprefa colla fcorta delle loro trombifte: ficchè perduto il di loro incaminamento, s' arreftano ingannate dal fuo. no del detto metallo, che vien da effe creduto il fufurrar appunto delle medefime trombifte, a detta del Falchini; oppure una campanella da fuonare, per avvifarle, che vi è a loro difpofizione una $\mathrm{Ca}$ a ; quefto fquillo da efse forfe creduto un tuono, a cui debba fuccedere qualche pericolofa tempefta, come fi ofserva nel Spetcacolo della IVacua : qual funno dee farfi vicino a qualfivoglia forta d'Albero, a cui più proflimo fia il fciame che fugge, perchè più facilmente fi fermino, e pofino fopra i di lui rami, come dice l'iftefso Falchini.

Fermate che fienfi, debbonfi tofto fpruzzar di Vi. no generofo, e così fare di quando in quando fino paf. fate le ore calde, perchè non intraprendino di nuovo la fuga; dalla quale poi, al dir del Falchini, farebbe dificile arreftarle: avvertendo, che, fe lo fciame è no. tabilmente divifo in due parti, bifogna porne una par. te in una Caffetta da per fe, e così l' altra parte in altra Cafsetta, perchè quefto è indizio, che in quella com. pagnìa vi fono due $\mathrm{Re}$, oppure ammazzare uno di effi Re; nondificile a difcernerf colla cognizione data nel primo Capo per porle, come a mio credere farebbe meglio, in un folo Alveare, maffime fe forsern poche; giac. chè foggiorna no infieme pacificamente, fin tanto che v'è fito, come ofservo nel detto Spettacolo della Natura. Circa poi le ore vintiquattro dello fteffo giorno, B 2 deefi 
deefi fpruzzar nuovamente le dette Api come fopra, tagliare tofto il ramo, e fcuoterle tutte ad un tempo nella nuova caffetta; ftrofinata prima al dì dentro con qualch erba odorifera, ovvero, non effendo poffibile tagliare il ramo, fottoporvi la caffeta, e fcuotervele con la mano inguantata, $o$, non potendofi fottoporvela, in tal Cafo ponervela al di fopra, e farvele entrar col profumo, di cui fi dirà nell' ultimo Capo; ivi lafciando Ja detta caffetta ben chiufa tutto il giorno feguente, coi bucchi però aperti, acciò poffino encrarvi ancora quelle che foffero cadute a terra, o in vicinanza difperfe, ad'unirfi con le loro compagne. Quindi il cuftode delle Api, paffato il detto giorno, e chiuf li bucchi di detta caffetta,l'afporterà agiatamente al luogo deftinarole, riaprendo li bucchi medefimi, come infegna if. Falchini anzidetto. Se poi ritornaffero a contraltare, debbonfi fpruzzar di polvere, perchè s'acquietino, co me vuole Virgilio nel mentovato lib. 4 .

Pulveris exigui jactu comprefsa quiefcent.

\section{$\begin{array}{llllll}C & \text { A } & \text { P } & \text { O } & \text { V. }\end{array}$}

Del tempo, e modo di levare i feli, e di arnmazzare le Api.

7 vero tempo di levare i fieli, a detta del Columella nel citato lib.9.cap. I 5. univerfalmente fi è, quando le Api fcacciano, ed uccidono li Pecchioni come inutiJi, e dannofi; il che fanno nel fine dell' Eftate, terminata che fia la generazione: Mox vere tran acto fequitur, ut dixi, melis vindemia, propter quam totius anni labar exercetup; ejus maturitas intelligitur, cum animadvertimus fucos ab Apibus expelli, ac fugari, quod eft genus. amplioris incrementi fimillimum Apis; ficchè cadendo it fine dell Eftate in quelti Paefi alli venti uno di Settembre, non fi dovranno levare li fieli, cioè le panne, dalle Caffette, prima di detto tempo; altrimenti, levandoli, il miele riefce troppo acquofo, a caufa dell ova inna- 
te, e delli vermicivoli non completi; in confeguenza anche di minor qualità, e pefo, come dall' efperienza confta.

Quanto al modo : provveduti che fieno li cuftodi delle Api, come dice il Falchini, d' un pajo guanti di Pelle, e d'un Cappuccio con gli occhj piccoli pur di Pelle, o di Panno da ripararfi le mani, e la tefta fin fot. to il corpetto ('ma per maggior ficurezza, e preftezza io direi effer meglio, che il Cappuccio foffe con gliocchj grandi di vetro concavo); di fumacchio della qualità che dirò, giufta le Figure C, D; d'una brenta da porre li fieli,

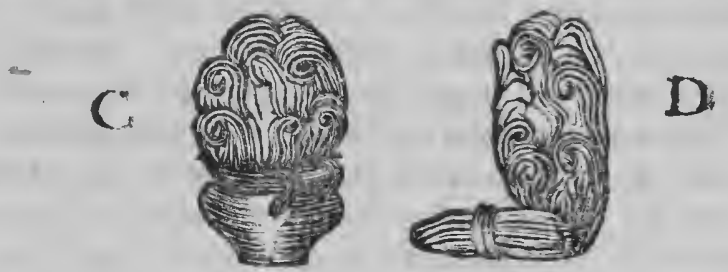

come la Figura $E$, d' un Coltello col taglio d' ambe le

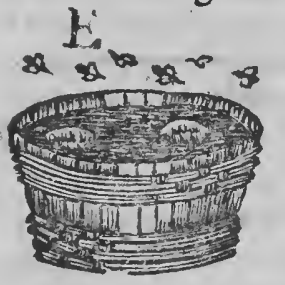
parti, e colla punta piana, e tagliente lungo quanto è alea la $\mathrm{Caf}$ fetta da levar i fieli, giufta la figura $F$; $e$ di un ferro in. zancato pur tagliente da radere ogni im. mondezza(vedi quila

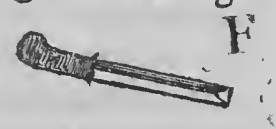

prefente Figura $G$;) come defcrive il Colummella nel G citato cap. is: Duobus ferramentis ad Cunis bunc ufum opus eft fexquipedali; vel paullo ampliore mensura factis, quorum alterum fit culter oblongus ex utraque parte acielata, uno capite aduncum babens fcalprum, alierum prima fronte planum, of acurifimum, quo melius boc favi fuccidantur, illo era. dantur, of quidquid fordium deciderit, attrabatur. Indi aperta e rivolta la Caffetta colla facciata a terra, e prefo il fumacchio accefo, debbono girarlo attorno ai fieli, che di mano in mano vorranno levare, perchè le Api a caufa del fumo ftieno lontane da chi opera, ed entrino frattanto in quei fieli nuovi, che vi dovranno lafciare B. 3 
per nutrimento di sì cari e utili animaletti fino all' er. be nuove; oppure, in vece del già detto riparo, fi co. prino bene la faccia, e le mani con denfo impiaftro fat to d'Oglio comune con polver di Fien Greco, e fugo di malve fllveftri, come infegna il citato Weckéronel det. to luogo: Ad farinam fani graci torrefacti malva fil. veftris fuccum affundito cum Oleo, factaque melis fpiffi. tudine, faciem, o nudas corporis partes largiter illise.

$S$ 'avverte però di lafciarvi la metà di ciò che vi fi troverà dentro, per non effer quefti Territorj così fertili d'erbe florifere come quelli d'altri Stati, ne' quali vi lafciano la quinta, o la fefta parte, fecondo che fono foggetti più o meno all. Inverno, giufta il documento dello fteffo Columella nel detto Capo is : atgue beic tamen modus non eft in omnibus regionibus certus, quoniam promultitudine florum, of ubertate pabuli Apibus consulendum eft; con offervare di levar la roba vecchia, la qual è fcura, lafciandovi la nuova ch'è gialla; e di far tut. ta quefta operazione fpeditamente, $e$ in ora frefca: dies vero caftrandi (fegue il medefimo Colrmalla) fori matutinus occupandus eft; neque enim convenit aftu medio exasperatas Apes lacesiri; poi chiudere tofto la Caffetta, riponendola a fuo fito come prima.

II Fumacchio più opportuno a quefto effetto, fi è di gomma galbana, perchè quefta non arde, ed'è afsai o. dorifera, oppure d'un pezzo di quelle facchette ad'ufo de Torcoli, nelle quali vi fieno ftate fpremute l'Ulive, alquanto fpruzzate d'acqua, perchè nè meno quefto farà fiamma, nè aftenderà le $A$ pi; o, in mancanza di efso, potrà fupplire il fumo di Arazze lana bagnate, o di fterco Bovino fecco; riguardandofi da qualunque altra forte di fumo, che non può efsere per le Apife non mortifero, come affermano il Falchini, ed il Rucellai ambi Scritcori Fiorentini.

Se poi vi folse qualche Cafsetta fcarfa di fieli per le difavventure, o, come vuole il Columella, per le rifse patite dalle Api con li Fucchi, cioè Pecchioni, in confeguenza fcarfí di provviggione per il futuro Inverno pon 
non dovranno levarne alcuno, ma lafciarla intatta, per non perdere le Api, che pur troppo colla fcarfezza del lavoro indicano aver patito; onde poffino alimentarfi, e rimetterí per l'anno feguente.

Quanto è biafimevole il coftume di quefti Paefi di ammazzare le Api ogni due, e tre anni, come forive anche il Tanara, con tanto difcapito nel ricavato dell' entrata proveniente dal loro lavoro; altrettanto è commendabile quello di non ammazzarle fe non ogni fei, o fette anni, come abbiamo nel Spettacolo della Natura Tom. I. Dialogo 6. e 7 , per la ragione, che, fatse vecchie, ficcome fciamano fempre le giovani, come accennai nel Cap. IV., in termine di detto tempo, le $\mathrm{Ca}$ merette, o Celette diventano molto angufte, cominciano a guaftarfi, e le Api non folamente s' infiacchifcono, ma il loro lavoro ancora diviene foggetio a'Vermi, ed alle Tignuole; le quali trovano il modo d' infinuarf in quelle Pelli, con cui i Vermiccivoli addobbano le mura della loro Cameretta, giufta l'ofservazione fatta dall'Accademia dell. Scinnze di Parigi: a vuerrendo in tal cafo di farle morire col zolfo, come nell'accennato Dia. logo 7. e non in alcra maniera, perchè il miele refti pith purgato, e di far quefta operazione in qualche diftanza dalla fituazione delle Api, per non apportarle nocumento.

Perciò non polso, nè debbo tralafciar di pregare, ed efortare chiunque ad' ufar quefta pratica facile, ed' utile afsai più di quella, che quì ingratamente fi ufa contro la vita di quefti Animaletti tanto amabili nella loro condotta, quanto nel lorolavoro, come fi ha nello fteflo Spettacolo della Natura, e contro il proprio interefse, come dall' efperienza d' ogni genere di perfone degli fopracitati Territorj, e dal non efservi alcun Autore che tratti in contrario. Per il che mi perfuado, che ognuno farà conto di quefta pratica, tanto più quanto che, levando ogn'anno la metà de i fieli, fi conleguifce ogni tre anni (oltre la confervazion delle Api) un terzo di entrata di più di quello che fi confeguirebbe coll' a mmaz- 
16

zarle, come qui malamente fi coltuma, e i fieli medef. mi incorrono anche minor pericolo di guaftarf, e le $A$. pi di ammalarfi a cagione della putrefazione del miele, di cui nell' ultimo capo fi tratterà .

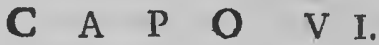

\section{Della maniera di cavar il miele, e la Cera:}

TEllo ftefso giorno, in cui fi levano i fieli dalle Caf. 1 fete, fi dee anche cavar fubito il Miele, finch' è tepido, e far quefta operazione al Sole nell'ora calda, perch' $e f c a$ più facilmente; pafsandolo in vafi di terra vetriati, o di legno ben netti, e fenz'alcun odore, mediante una graticola di ferro, rame, o altro, fatta a guifa di colatojo; fopra la quale fi pofino e fcolino quafi di per sè tutt'i fieli, a rifserva diquelli che fofsero guafti. Quefto primo miele è il più perfetto, e fi chiama Miel Vergine.

Scolati she fienn, come fonra. li detti Goli, filebho: no porre in un facchetto di tela di canape fimile a quelli, coi quali fi colano i Vini, e premere con due bano. ni lifci, finchè $f_{1}$ veda ufcire a forza il miele. Quefto per efser così fpremuto, è molto men perfetto del primo; perciò dee porfi feparato in altri vafi fimili, e vien detto Miele di frucco.

Tutti quefti Vafi poi debbonfi lafciare áperti qual. che gicrno, affinchè il miele fi purghi, anzi, prima di chiuderli, bifogna levarli la fchiuma, che di mano in mano andrà facendo, per poterlo ferbar in detti Vafi purgato da ogni immondezza all' ufo opportuno; fervendo quefto, giufta il parer del Rucellai, a mantener an. che incorrutibili molte frutta, che vi fi immergeffero, ed a condirle, fe foffero acerbe:

$$
\begin{aligned}
& \text { Atti a dolcir con esso acerbe frutta } \\
& \text { Nespoli, e forbe, e l'agro Umor dell' Uva. }
\end{aligned}
$$

come nelle fue ofservazioni dice venir praticato partis colarmente a Napoli.

Final. 
Finalmente dopolevato il miele nella forma già detta; fi debbono prima lavar i fielicon acqua pura,e porre in una Caldaja di rame pulita: dentro la quale (già coperti di altra acqua netta) bollino per lo fpazio d'un ora circa, affin. chè la Cera refti purgata; levando frattanto con mineftro forrato l'immondezze che vengono a gala dell' acqua bol. lente.

Indi fi faccia paffare tutto ciò che refta in detta Calda. ja , per un altro facchetto fimile, e, ripulita la Caldaja, pongafi dinuovoal fuoco con dentro un terzo d'acqua, $e$ due di Cera, e, toftocchè quefta fia ben liquefatta $e$ pulita, fi vuota in Vafi larghi fpruzzati d'acqua, affinchè non vi fi attacchi, per ridurla in formelle da vendita. Fin quì l'antecitato Falchini.

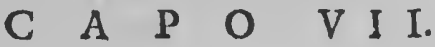

Delle piu neceffarie avvertenze per $i$ Cuftodi, - per la prefervazione delle Api.

Tce Palladio riportato nel principio delle annotazioni al Kucellai, che le Api pungono acerbamente quelli che vi fi accoftano macchiati d'impurità : onde lafciò fcritto purus cuftos frequens oo caftus accedat, come anche fcriffe Virgilio nel fopradetto lib. 4 .

Illum adeo placuiffe Apibus mirabere morem,

Quod nec concubitu indulgent, nec corpora fegnes,

In Venerem Solvunt.

e però fi regolino i Cuftodi a contenerfi, fe bramano ri. cornarfene dalla vifita illefi.

Patifcono ancora la rabbia in modo particolare, quando le vien fatto qualche oltraggio, ficchè $s^{\prime}$ adiraro talmente, che loro efce dalla bocca (al dir del predetto $\mathrm{Ru}$ cellai) un certo vapor nero, che fi alza per aria a guifa di picciola nube; ed' allora è, che la lor puntura è molto pericolofa:

Allora ì il morso lor rabbioso, e infetto, $E$ si mortal velen le infiamma il cuore, 


\section{Che le ciecbe faette entr' alle piagbe \\ Lafciano infiff con la vita infleme.}

Perciò offervi bene i! Cuftode maggiormente quì il fatto fuo, per non accoftarvifi durante tal rabido loro umore, o, dovendovifi accoftare, fpruzzarle prima di Vin puro, fintantocchè fianfi mitigate .

Al dire delli fopracitati ScrittoriFiorentini, le A pi non fi ammalano fe non per mancanza di vito; per la putrefa. zione del miele canata dalle Farfalle, e dalli Cacchioni che fono le Api innate, e per la peftilenza, alla quale fono foggetti tutti gli Animali a caufa della diverfità degli tumori.

Quanto alla prima, debbono i loro Cultodi tenerle prov. vedute fino alli nuovi fiori di miele, o in mancanza dique. fto, di Ficchi fecchi, Uve, o melefecche cote prima nell' acqua, ponendole attorno alle $\mathrm{C}$ affette per loro alimento.

Riguardo alla putrefazione, debbono levare deftramente con il coltello defcritto nel Cap. 5. li fieli guafti fenza toc. care lifani, e profumarle leggermente con Timo, Centaurea, Santoreggia, volgarmente detta Salezola, o altr'erbe odorifere focolo, affonchè c' invignrifrano, o rifanino, oppure coll'Incenfo, come infegna il Falchini.

Rifpetto poi alla peftilenza, ch'è come una fpezie di dierea, detta volgarmente rilaffo di corpo, alla quale fono foggette maffine a cagione delli fiori d'Olmi, e Titimali detti dal Volgo lattaroli, che diffolvono i corpi loro, come fcrive il Rucellai :

\section{Quefto con chiari Jegni ti fia noto}

Mafimamente in ful forir de l'olmo,

o del verde Titimalo, che folve

$I$ corpi lor, come $S$ camosio, $i$ nofri.

e prima di lui il Columella nell' antecitato lib. 9. cap. I3. ove dice: Maximus autem annuus earum labor eft initio veris, quo Titbimali floret frutex, oo quo Sameram Ulmi pro. munt; namque, ficut novis pomis, it a bis primitivis floribus illecte avide vefcuntur poft bybernam famem, nil alioguin citra fatietatem, tali nocit te cibo, quo, cum fe affatim replenerint, profuvio alui, nifs celeriter fuccurritur, interiunt,

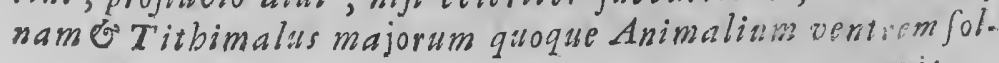


Sopra la cultura delle Api.

vit, of proprie Ulmus Apium, eaque caufa eft, cur in Regio. nibus ltalia, qua funt ejus generis arboribus corfita, raro frequentes durent Apes.

A quefta malattia debbono li Cuftodi tofto rimediare, affinchè non muojano, col porre preffo l'Alveare infetto qualche vafo di molto cotto, o di perfetto,e fpiritofo Vino dolce, ovvero di miele bollito in due porzioni d'acqua con galeghe,o rore fecche; foppraponendovi un velo, o lia pezza di lana, o di tela bianca, acciò le Api vi fi fermino, e non poffino affogarfi ; indicon il fumacchio accefo, fugge. rito nel Cap. 5., con odori, e profumi farle ufcir fuori dalli fieli a fucchiare il detto liquore per rifauarle: boc modo, cosł Plinio nel lib. delle A pi citato dal Columella fuddetto, Succurrendum laborantibus exifimat.

Per tali motivi fi rende neceffario vifitarle di quando in quando dalli primi di Febbrajo per tutto Maggio, coffer vare fe vi fieno fieli in qualche parte corrofi, effendo que. fto un indizio certo, che le Api fono prive di nutrimento; oppure fe ve ne fieno con alcune macchie nere fcure,ch'e appunto un vero conus $x_{\text {guu di }}$ rere gualto il miele; finalmente $r_{c}$ vi fieno Api tramortite dolenti e mefte, ch'è fegno infallibile del già detto morbo, come afferma il Rucellai:

Allor fi fente un Sufsurar piit grave

Fra loro, e un fuono dolorofo, e mefto,

Allor le vedi impallidirs in volto,

$E$ farfi eftenuate, orride, e secche.

Non folamente nelli detti quattro mefi deve il Guardiano liberare le Api dalle fopradette infermità, e infezioni, ma ancora nelli quattro altri feguenti deve levare dalle Caffette li Ragni, che colle loro tele impedifcono il bel la. yoro del miele, e tener fgombro, cioè libero tutto l'interno delle medefime Caffette da ogni erba, e immondezza, perchè non vi fi annidi alcuna fpezie d'Animali; difenden. dole fopra tutto dalli Calabroni, e dalle Farfalle più nocivi alle Api d'ognialtro Animale, come avvertono li fud. detti Scrittori Fiorentini.

Quefti Calabroni fono di due forte: i primi del color delle 
delle Api, che fi chiamano dal volgo Martinelloni, o fia Mattoncini groffi, da' quali vengono uccife; i fecondi di color nero detti gralaoni, quali entrano nelle Caffette a fucchiare il miele, e tal volta mangiano anche le Api che fono nelle loro Cellette. Per le quali cofe ognuno vede quanto fia neceffario a mmazzarli fenz' altra racoman. dazione, mentre non poffono le Api liberarfene di per fe, avendo quefti la cute duriffima.

Per diftruggere pofcia le Farfale, of a Poveglie(che fono del miele la pefte maggiore, come afferifce Eliano nel lib. r. della Storia degliAnimali, dalle quali fi producono le Ti. gnuole, che ne deriva polcia la putrefazione predetta) convien che il Cuftode delle A pi le prenda per mezzo d'un vafo grande fenza fondo, largo nel piede, e ftretto nella ci. ma con ivi un foro, da cui polfa efalar il vapore, o fia un imbuto da vino, volgarméte lora, con entro un lume fin quafi a terra; ponendo quefto vafo la fera fra le malve fiorite; nel qual tempo quefti Infetti fogliono in effe più germogliare ;oppure preflo gli Alveari, che in poche ore (tratte dal lume in effo valo per uecuu roru, le avrà da fe fteffe imprigionate, e morte. Del che fe ne aflicura il citato Rucellai, feguendo l' autorità del Columella, il quale, parlando delle Urne di que' tempi, fcriffe nell' antedetto lib. 9. cap. 14. Quo tempore malva florent, cum eft earum maxima multitudo, fo vas aneum fimile miliaro vefpere ponatur inter alvos, os in fundum ejus lumen aliqnod di. mittatur con quel che fegue.

Un' altro rimedio del Rucellai fuggerifco, per allontanare non folo le dette Farfale, ma ancora le Racole, e Formigoni alati, li Mofconi, ed i Scorpioni col fugo dell' Appio, cioè del feleno felvatico, e del Petrofello co. munemente detto Perfemolo, o col fugo dell' Origano fflveftre, detto volgarmente Puleggio dai fiori pavonazzi; ftrofinando con detro fugo la facciata delle Cafsette, men. tre l'odor di quefto li fa ritrarre dalle medefime.

Per animar finalmente ogn' uno a porre in pratica sì fatte offervazioni, e cautele, voglio aggiungere, che in çuefi Paefi ( per quanto vengo afticurato da perfone de- 


$$
\text { OJervaz. Sopra la cultura delle Api". }
$$

gnè di fede) mezzo Alveare d'Api può rendere annual. mente per lo meno due pefi di panne, le quali, purgandole, come fi detto, Idaranno almeno quaranta lire di Miele, due terzi Vergine, ed un terzodi Strucco, e quattro lire Cera di formella; del che facendone la pruova, ne ve. drà l' effetto, purchè le ftagioni fieno temperate.

Molte altre cofe intorno alla coltivazione delle A pi a$v$ rei potuto dire; $m a$, ficcome mia intenzione fu di dar folamente alla luce le principali e dal Falchini con Ia lun. ga efperienza ofservate, e dall Accademia delle Scienze

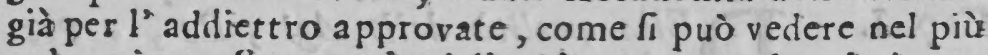
volte citato Spetracolo della Natura, e da altri ancora in quefte mie Ofservazioni rapportate; così non mi fono molto dilungato in tale materia, baftandomi di aver fuccintamente detto quel tanto, che vaglia ad' il. luminar il buon Cultore, e nello ftefso tempo a levar quegli abufi fopra tale coltivazione, pe quali ritrare non potevafi quel vantaggio, che coll'ufo di tali Ofserva. zioni infallibilmente fo ritrarrà. Se quefta mia fatica, la quale a folo pubblico benefizio ho intraprefa, verrà a buon grado ricevuta, in me fi accrefcerà fempre più il corag. gio d indagare con continue ofservazioni i fecreti di quel. le cofe, le quali fono più in ufo per la coltivazione, nella quale e ditetto, e utilità fomma ne ricavo:

\section{IL FINE.}




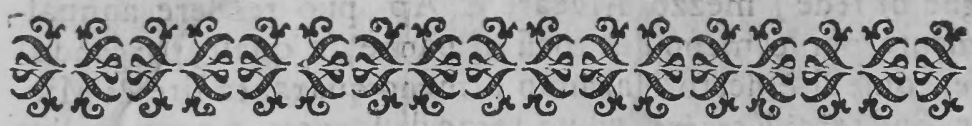

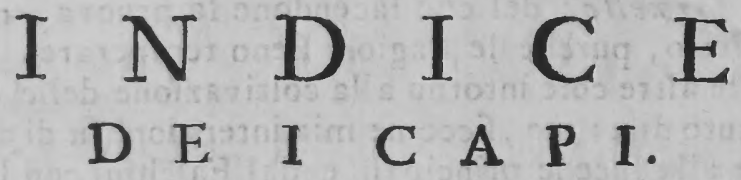

Cap. I. Della qualità delle Api, $R_{e}$, e Peccbioni. pag. I.

Cap. II. Dell'opportuna fituazione; e paftura per le Api.

Cap. III. Degli Alveari, o fiero Caffette per le Api, tempo di provvederle, e modo di trasportarle.

Cap. IV. Della vera maniera di trattenere, e pres. dere le Api, cbe fciamano. 10

Cap. V. Del tempo, e modo di levare i fieli e di ammazzare le Api. 0.12

Cap. VI. Della maniera di cavar il miele, e la Cera. $\quad 16$

Cap. VII. Delle pii neceffarie avertenze per $i C_{u-}$ Aodi, e per la preservazione delle Api. I7. 
$(c) b z 2$.
leate

fonen?

$$
\begin{aligned}
& \text { fipune oexte } \\
& \text { Intecenoter e rous } \\
& \text { Perpots ante }
\end{aligned}
$$

